\title{
The Eco-evolutionary Modelling of Populations and their Traits using a Measure of Trait Differentiation
}

\author{
Roger Cropp \\ School of Environment and Science, Griffith University, Gold Coast, Qld, 4215, Australia \\ Centre for Applications in Natural Resource Mathematics, School of Mathematics and \\ Physics, The University of Queensland, St Lucia, Qld 4072 \\ John Norbury \\ Mathematical Institute, University of Oxford, Andrew Wiles Building, ROQ, Woodstock \\ Road, Oxford OX2 6GG, UK
}

\begin{abstract}
We develop new equations for the eco-evolutionary dynamics of populations and their traits. These equations resolve the change in the phenotypic differentiation within a population, which better estimates how the variance of the trait distribution changes. We note that traits may be bounded, assume they may be described by beta distributions with small variances, and develop a coupled ordinary differential equation system to describe the dynamics of the total population, the mean trait value, and a measure of phenotype differentiation. The variance of the trait in the population is calculated from its mean and the population's phenotype differentiation.

We consider an example of two competing plant populations to demonstrate the efficacy of the new approach. Each population may trade-off its growth rate against its susceptibility to direct competition from the other population. We create two models of this system: a population model based on our new ecoevolutionary equations; and a phenotype model, in which the growth or demise of each fraction of each population with a defined phenotype is simulated as it interacts with a shared limiting resource and its competing phenotypes and populations.

Comparison of four simulation scenarios reveals excellent agreement between
\end{abstract}

Preprint submitted to Journal of Theoretical Biology

August 27, 2021 
the predicted quantities from both models: total populations, the average trait values, the trait variances, and the degree of phenotypic differentiation within each population. In each of the four scenarios simulated, three of which are initially subject to competitive exclusion in the absence of evolution, the populations adapt to coexist. One population maximises growth and dominates, while the other minimises competitive losses. These simulations suggest that our new eco-evolutionary equations may provide an excellent approximation to phenotype changes in populations.

Keywords: Eco-evolutionary model, trait adaptation, beta distribution, trait differentiation

2010 MSC: 00-01, 99-00

\section{Introduction}

The role of eco-evolutionary processes, in which population dynamics and adaptation occur on similar time scales, has long been recognised in many biological systems, with a variety of theoretical approaches applied to understand 5 the influence of these processes [39, 21]. The current rapidly changing climate of the Earth, with its impact on species' thermal tolerances, ranges, habitats and phenologies, indicates a pressing need to develop eco-evolutionary models that can predict how populations, species and ecosystems will adapt to climate change [57. Despite the abundance of theoretical approaches, it appears to remain the case that much of our ability to predict the impact of climate change on ecosystems stems from empirical observations of how they have responded to past change 42 .

Many eco-evolutionary approaches assume that traits/phenotypes are normally distributed within a population, and early derivations of equations describing the evolution of traits often relied on assumptions of normally distributed traits [30. However, recent empirical evidence suggests that some traits may have non-normal distributions [26], and theoretical considerations suggest that most traits will have bounds [12, 25]. Although normal distributions may 
be effectively bounded in simulations [52, a more useful approach may be to assume traits are described by other distributions that are intrinsically bounded [52, 27]. Beta distributions are particularly useful in trait simulations, as they may be symmetric, skewed, or bimodal, whereas a normal distribution is always symmetric and unimodal.

Evolutionary bounds may stem from physical considerations such as maximum or minimum body size, as Kempes et al. 25. suggest, environmental tolerances, or population traits that, for example, may limit mutualism benefits as Cropp and Norbury [11, 13] have shown theoretically. The use of a bounded trait distribution such as a beta distribution has the advantage of allowing phenotypes to explore the bounds of a trait, and for eco-evolutionary so equilibrium points at the bounds to potentially provide useful information on the eco-evolutionary system dynamics. We do not argue that all trait distributions are bounded, indeed our ecological framework explicitly shows that some have no theoretical bound (although they may be effectively bounded by physiology, etc). In this work we focus solely on bounded traits.

Ecological models have provided the cornerstone of ecological theory for much of the past century, from the classic Lotka-Volterra models [37, 56] of predation and competition through to simple, but disruptive, models that revealed the potential for chaos in living systems [38, 22]. Ecological models perforce make assumptions about ecological interactions (i.e. who eats whom, and how

40 fast) that apply to both phenotypes and populations. These assumptions define life functions that give the net per capita growth rate of a population or phenotype. The abundances of the populations or phenotypes wax or wane according to their life function.

Eco-evolutionary models are extensions of ecological models that may be 45 considered population models or phenotype models, and we shall use this terminology in this paper. Population models simulate the average population dynamics (the "zeroth" moment of the trait distribution), the mean trait value (the first moment), and sometimes the trait variance (the second moment) of the populations $[21,28$. Phenotype models, sometimes called full trait distribu- 
tion models, simulate the dynamics of individual phenotypes, each of which is identified by a different trait value [47, 50, 15, 27, 49], and so simulate the entire trait distribution, from which all the moments of the trait distribution may be calculated. The population size, trait mean and trait variance may be derived from the phenotype abundances for comparison with an equivalent population 55 model.

There are then two important differences between phenotype models and population models. Phenotype models do not make assumptions about trait distributions, but simulate the full trait distribution from which all the moments of the trait distribution may be calculated. Population models do make assumptions about the trait distributions, and simulate only a small subset of the trait distribution moments.

Eco-evolutionary population models generally assume that traits have a particular functional form for the distribution, often normal [31] or beta [27, that becomes a fundamental attribute of the model. Phenotype models do not require assumptions to be made about how traits are distributed. The initial conditions used to start phenotype model simulations include a distribution of phenotypes chosen by the modeller, but this does not impose a particular form of distribution on the model, as in cases where the model dynamics are not critically dependent on the initial conditions, the influence of the initial conditions 70 is removed through the transient dynamics of the system.

Eco-evolutionary population models describe how the average trait value of a population evolves [31, 23], and so also define what determines the average fitness of the population [19, $18,8,40$. They use the sensitivity of the average fitness to the trait to determine how the average trait value of the population changes. The average per capita growth rate is often used as a natural measure of fitness. Eco-evolutionary population models may assume that the variances of trait distributions are constant, for example Lande [31], but may include equations that describe how the variances of the trait distributions change, for example Klauschies et al. 27].

A useful approach to test the veracity of eco-evolutionary population models, 
that simulate entire populations and their average trait properties, for example Abrams and Matsuda 4], Abrams [2, Cortez 9], is to compare their predictions to those of models that simulate each individual phenotype [28, for example Coutinho et al. 10], Klauschies et al. [27], Cropp and Norbury [14]. Phenotype models are complicated and usually defy analysis, often requiring long and extensive computations to reveal their properties, and hence are of limited use in understanding or predicting the outcomes of evolutionary processes in ecosystems without extensive and detailed post-processing of large amounts of data. However, their advantage of incorporating fewer assumptions than population models makes them a more reliable predictor of eco-evolutionary outcomes.

The population and phenotype approaches may be related by moment-based expansions for the populations, and the means and variances of the trait distributions, that may be closed in the case of non-normal distributions using the skewness and kurtosis of the trait distribution [41, 10, 27]. We adopt a similar approach here, together with a small variance limit, but we use different expressions for the skewness and kurtosis. We compare the results of simulations of a simple, but computationally demanding, phenotype model with those of a much less computationally-intensive eco-evolutionary population model that assumes that the traits have beta distributions with small variances.

${ }_{100}$ We take a novel approach to develop the equations governing the evolutionary dynamics of the population model. We derive equations describing how the total population and the mean and variance of its trait change, as similar models do. However, instead of an equation that describes directly how the variance changes, we model how the shape of the distribution of the phenotypes within the population (the "phenotypic differentiation") changes and calculate the variance from that. Phenotypic differentiation reflects the differentiation of allele frequencies between sub-populations, and in populations where phenotypic adaptation is not affected by the environment (as we consider here), it mirrors genetic differentiation [48]. Phenotypic differentiation indices, which may be evaluated at the trait or locus level, can be used as indicators of the extent of adaptation in natural populations [35, 51]. 
Phenotypic differentiation is an analogous measure to the fixation index $F_{S T}$ [61], and may be calculated in a similar manner [48. While fixation indices are defined by ratios of allele variances in sub-populations and populations, phenotypic differentiation uses ratios of phenotype variances. The relationship between the fixation index and phenotypic differentiation in a population is commonly used as an indicator of selection pressures [59. We use phenotypic differentiation as a guide to develop an analogous variable to simulate evolutionary processes that influence trait variance in eco-evolutionary models, as described in $\$ 2.3$

This approach of using an analogue of phenotypic differentiation rather than calculating variance directly provides well-behaved eco-evolutionary equations that allow the trait means to evolve all the way to the boundaries of their distributions. The equations are of closed form, that do not require further or external information to be solved, and do not contain singularities at the bounds that arise in some other approaches, for example Klauschies et al. 27, Yamamichi et al. 62. Numerical simulations reveal extremely good agreement between this population model and the equivalent phenotype model under the small variance approximation.

\section{Theoretical Support}

The theoretical basis for the modelling approach outlined below rests on some key assumptions. Both phenotype and population models include the ecological conditions that:

- the abundances of resources, populations, and phenotypes are represented in a common, conserved currency; and

- each population, or phenotype within a population, must have a resource that it depends upon for survival explicitly modelled in the system; and

- a population or phenotype must be able to grow when its resource is maximal, and must be unable to grow when its resource is zero. 
The theoretical basis for the evolutionary component of the population modelling approach outlined below includes the assumptions that:

- the fitness of a population is reflected by its net per capita growth rate function, the small variance limit of the phenotype per capita growth rate function;

- the beta distribution with small variance may be used as a convenient representation of the distribution of phenotypes with different trait values in populations; and

- the extent of phenotypic differentiation, and hence trait variance, within a population provides a useful metric to follow how the shape of the distribution of trait values changes over time.

The beta distribution is commonly used in the absence of data identifying a particular distribution [32. It can produce a wide variety of shapes within a finite interval [24], and can be related to other distributions such as uniform, $\chi^{2}$, gamma and $F$ 45, as well as approximating a Gaussian distribution. The standard beta distribution is defined on the interval $0 \leq x \leq 1$ and its probability density function is given by:

$$
f_{\beta}(x ; \alpha, \beta)=\frac{x^{\alpha-1}(1-x)^{\beta-1}}{B(\alpha, \beta)}, \quad \text { where } B(\alpha, \beta)=\frac{\Gamma(\alpha) \Gamma(\beta)}{\Gamma(\alpha+\beta)},
$$

and $\Gamma$ is the gamma function. The exponents $\alpha>0$ and $\beta>0$ are parameters that control the shape of the beta distribution. The mean of a beta distribution is given by $\alpha /(\alpha+\beta)$, and the variance by $\alpha \beta /\left[(\alpha+\beta)^{2}(\alpha+\beta+1)\right]$.

We note that eco-evolutionary models may be formulated in many different ways 21, 28, and may assume bounded (for example, beta) or unbounded (for example, normal) trait distributions. Trait values are defined to be positive, and in 2.1 we show that basic ecological principles may place additional constraints on the values that traits may take. As there are often simple ways that ecoevolutionary models may be written so that bounded distributions such as beta may be incorporated, we do not consider the assumption of a beta distribution to 
be any more restrictive than the common assumption of a normal distribution. We test the validity of the beta assumption by comparing the output of the population model to that of the phenotype model which makes no assumptions about the distributions of traits.

\subsection{Ecology}

The ecological basis for the modelling is set out in detail in Cropp and Norbury [11, so we include here just a brief synopsis of its key properties and their implications for eco-evolutionary modelling. Essentially, the modelling framework requires populations in an ecosystem to be modelled by ordinary differential equations of Kolmogorov form [29]. For clarity, here we will discuss only populations, but the description might equally be applied to phenotypes within populations:

$$
\frac{d P_{i}}{d t}=P_{i} f_{i}\left(P_{1}, P_{2}, \ldots, P_{m} ; N, \Omega\right), \quad i=1,2, \ldots, m,
$$

where $P_{i}$ is the scaled population measure, $N$ is the scaled nonliving resource pool measure, $\Omega$ are parameters that describe the attributes of the population (i.e. feeding and mortality rates, etc), and $m$ is the number of populations. The function $f_{i}$ of these quantities describes the net per capita rate of change of the population $P_{i}$. The populations and resources are measured in a common currency of the sole limiting resource, that is often a nutrient. Note that the ecological modelling framework we describe can apply to populations, subpopulations, or phenotypes.

Equation (2) represents a system that is potentially open to flows of energy and mass but is closed to flows of the limiting nutrient, so that $P_{1}+P_{2}+\cdots+P_{m}+$ $N=1$ ( $1=100 \%$ of the cycling limiting nutrient in the food web by choosing appropriate units of measurement for the ecosystem under study). Approximate closure with respect to the mass of the limiting nutrient is a common property of ecosystems [55], and exact closure has the advantage that an ordinary differential equation to represent the change in $N$ is not required. Note that while the 
resource for many populations in 2 will be another population, at least one population must be an autotroph that feeds upon the nonliving resource $N$.

Simple conditions are placed upon the $f_{i}$ in equation (2) to ensure that only realistic ecosystem models are considered. Realistic ecosystems do not contain unviable or immortal populations, that is, a population must be able to grow when its resource is maximal, and must be unable to grow when its resource is zero. An autotroph population $P_{i}$ that feeds upon resource $N$ is constrained to those populations that meet the conditions $\left.f_{i}\right|_{N=1}>0>\left.f_{i}\right|_{N=0}$. For example, consider a simple nutrient-dependent Michaelis-Menten growth term $f_{i}=\mu N /(N+\kappa)$. Satisfying the condition $\left.f_{i}\right|_{N=1}>0$ requires that $\mu>0$ and $\kappa>-1$; as negative half-saturation constants have no biological meaning the effective constraint is $\kappa>0$.

The conditions imposed upon population parameter values (equivalently traits) require that many, if not most (depending upon how the model is written), evolving traits are subject to one and sometimes two bounds on the parameter values they can take. These evolutionary bounds are explicitly set by the ecological properties of the model, and imply that assumptions of bounded distributions for population traits or phenotypes are, perhaps in general, more appropriate than assumptions of unbounded distributions.

\subsection{Evolution}

We assume that phenotypes are distinguishable by a continuously varying trait in an otherwise homogeneous population. Here we describe a single trait in a single population $P$, so that we may drop the subscripts used in equation (2) for clarity of notation, but the equations we derive may be extended to ecosystems with multiple interacting populations that each may have multiple evolving traits.

The trait is measured by the trait parameter $\gamma$, a member of the set of parameters $\Omega$, the values of which have been scaled (by choice of the unit of trait parameter measurement) to lie in the interval $0 \leq \gamma \leq 1$. Phenotypes in the population are identified by their $\gamma$ value. In the following we shall not refer 
to individual phenotypes, as the eco-evolutionary models do not resolve these,

$$
\frac{1}{P} \frac{d P}{d t}=\left.f\right|_{\gamma=\hat{\gamma}}+\left.\frac{v}{2} \frac{\partial^{2} f}{\partial \gamma^{2}}\right|_{\gamma=\hat{\gamma}}+\left.\frac{1}{6} M_{3} \frac{\partial^{3} f}{\partial \gamma^{3}}\right|_{\gamma=\hat{\gamma}}+\left.\frac{1}{24} M_{4} \frac{\partial^{4} f}{\partial \gamma^{4}}\right|_{\gamma=\hat{\gamma}},
$$

where the moments $M_{3}=S v^{3 / 2}$, and $M_{4}=K v^{2}$, for $v$ the variance, $S$ the skewness, and $K$ the kurtosis. The mean $\hat{\gamma}(t)$ of the trait $\gamma \in(0,1)$ satisfies:

$$
\frac{d \hat{\gamma}}{d t}=\left.v \frac{\partial f}{\partial \gamma}\right|_{\gamma=\hat{\gamma}}+\left.\frac{1}{2} M_{3} \frac{\partial^{2} f}{\partial \gamma^{2}}\right|_{\gamma=\hat{\gamma}}+\left.\frac{1}{6} M_{4} \frac{\partial^{3} f}{\partial \gamma^{3}}\right|_{\gamma=\hat{\gamma}},
$$

and the variance $v(t)$ :

$$
\frac{d v}{d t}=\left.M_{3} \frac{\partial f}{\partial \gamma}\right|_{\gamma=\hat{\gamma}}+\left.\frac{1}{2}\left(M_{4}-v^{2}\right) \frac{\partial^{2} f}{\partial \gamma^{2}}\right|_{\gamma=\hat{\gamma}}+\left.\frac{1}{6}\left(M_{5}-v M_{3}\right) \frac{\partial^{3} f}{\partial \gamma^{3}}\right|_{\gamma=\hat{\gamma}}+\left.\frac{1}{24}\left(M_{6}-v M_{4}\right) \frac{\partial^{4} f}{\partial \gamma^{4}}\right|_{\gamma=\hat{\gamma}} .
$$

We assume further that the population is distributed amongst its traits according to a beta distribution with parameters $\alpha(t)$ and $\beta(t)$ for all $t>0$ :

$$
P(\gamma, \alpha, \beta)=P(t) \frac{\gamma^{\alpha-1}(1-\gamma)^{\beta-1}}{B(\alpha, \beta)}
$$


The general expansions (3), (4), and (5) are re-expanded as follows to second order in the small variance $v>0$, first noting that:

$$
M_{3}=\frac{2(1-2 \hat{\gamma}) v^{2}}{\hat{\gamma}(1-\hat{\gamma})+v}=\frac{2(1-2 \hat{\gamma})}{\hat{\gamma}(1-\hat{\gamma})} v^{2}+O\left(v^{3}\right) \text { as } v \rightarrow 0 .
$$

Note $S^{2}=M_{3}^{2} / v^{3}=\frac{4(1-2 \hat{\gamma})^{2}}{\hat{\gamma}^{2}(1-\hat{\gamma})^{2}} v+O\left(v^{2}\right)$ as $v \rightarrow 0$. Then

$$
\begin{aligned}
M_{4} & =\left\{\left(\frac{\hat{\gamma}(1-\hat{\gamma})-v}{2 \hat{\gamma}(1-\hat{\gamma})+4 v}+1\right) S^{2}-\frac{6 v}{\hat{\gamma}(1-\hat{\gamma})+2 v}+3\right\} v^{2}, \\
& =3 v^{2}+\frac{6\left(1-5 \hat{\gamma}+5 \hat{\gamma}^{2}\right)}{\hat{\gamma}^{2}(1-\hat{\gamma})^{2}} v^{3}+O\left(v^{4}\right) \quad \text { as } v \rightarrow 0 .
\end{aligned}
$$

Then (3), (4), and (5) become, as $v \rightarrow 0$ :

$$
\begin{aligned}
\frac{1}{P} \frac{d P}{d t}= & \left.f\right|_{\gamma=\hat{\gamma}}+\left.\frac{v}{2} \frac{\partial^{2} f}{\partial \gamma^{2}}\right|_{\gamma=\hat{\gamma}}+\left.\frac{1}{3} \frac{(1-2 \hat{\gamma})}{\hat{\gamma}(1-\hat{\gamma})} v^{2} \frac{\partial^{3} f}{\partial \gamma^{3}}\right|_{\gamma=\hat{\gamma}}+\left.\frac{1}{8} v^{2} \frac{\partial^{4} f}{\partial \gamma^{4}}\right|_{\gamma=\hat{\gamma}}+O\left(v^{3}\right), \\
\frac{d \hat{\gamma}}{d t}= & \left.v \frac{\partial f}{\partial \gamma}\right|_{\gamma=\hat{\gamma}}+\left.\frac{(1-2 \hat{\gamma})}{\hat{\gamma}(1-\hat{\gamma})} v^{2} \frac{\partial^{2} f}{\partial \gamma^{2}}\right|_{\gamma=\hat{\gamma}}+\left.\frac{1}{2} v^{2} \frac{\partial^{3} f}{\partial \gamma^{3}}\right|_{\gamma=\hat{\gamma}}+O\left(v^{3}\right), \\
\frac{d v}{d t}= & \left.\frac{2(1-2 \hat{\gamma})}{\hat{\gamma}(1-\hat{\gamma})+v} v^{2} \frac{\partial f}{\partial \gamma}\right|_{\gamma=\hat{\gamma}}+\left.\left(1+\frac{6\left(1-5 \hat{\gamma}+5 \hat{\gamma}^{2}\right)}{\hat{\gamma}^{2}(1-\hat{\gamma})^{2}} v\right) v^{2} \frac{\partial^{2} f}{\partial \gamma^{2}}\right|_{\gamma=\hat{\gamma}} \\
& +\left.\frac{1}{6}\left(M_{5}-\frac{2(1-2 \hat{\gamma})}{\hat{\gamma}(1-\hat{\gamma})} v^{3}\right) \frac{\partial^{3} f}{\partial \gamma^{3}}\right|_{\gamma=\hat{\gamma}}+\left.\frac{1}{24}\left(M_{6}-3 v^{3}\right) \frac{\partial^{4} f}{\partial \gamma^{4}}\right|_{\gamma=\hat{\gamma}}+O\left(v^{4}\right) .
\end{aligned}
$$

We want expressions that are valid for long times, in particular, the evolutionary time scale that is approximately the inverse of the variance scale. For the population and trait mean equations, one power of $v$ on the right hand side does this to leading order. Since the variance is assumed to be small, we need two powers of $v$ on the right hand side in the variance equation. So our leading order model valid on the longer evolutionary time scale is:

$$
\begin{aligned}
\frac{1}{P} \frac{d P}{d t} & =\left.f\right|_{\gamma=\hat{\gamma}}+\left.\frac{v}{2} \frac{\partial^{2} f}{\partial \gamma^{2}}\right|_{\gamma=\hat{\gamma}}, \\
\frac{d \hat{\gamma}}{d t} & =\left.v \frac{\partial f}{\partial \gamma}\right|_{\gamma=\hat{\gamma}}, \\
\frac{d v}{d t} & =\left.\frac{2(1-2 \hat{\gamma})}{\hat{\gamma}(1-\hat{\gamma})} v^{2} \frac{\partial f}{\partial \gamma}\right|_{\gamma=\hat{\gamma}}+\left.v^{2} \frac{\partial^{2} f}{\partial \gamma^{2}}\right|_{\gamma=\hat{\gamma}}
\end{aligned}
$$


The per capita change in an evolving population then depends upon the ineach with multiple traits.

Analogously to the most commonly used computational form for $F_{S T}$ 61, 35, 58, we define $Q$ from the variance $v$ to be:

$$
Q:=\frac{v}{\hat{\gamma}(1-\hat{\gamma})} .
$$

Note that the Balding and Nichols [5] model, which is the basis for much forensic DNA analysis, defines $F_{S T}=1 /(\alpha+\beta+1)$, where $\alpha$ and $\beta$ are the shape parameters of the beta distribution. The variance of the beta distribution in 
equation (11), which follows Spitze's analogy of $Q_{S T}$ for phenotypes [51] to Wright's $F_{S T}$ for genotypes [60, then defines $Q=1 /(\alpha+\beta+1)$. This may be obtained algebraically from (11) in the case of a beta distribution.

$Q$ is representative of the differentiation between phenotypes within the population. $\alpha \approx 0 \approx \beta \Rightarrow Q \approx 1$ represents maximum differentiation between phenotypes. In this case, the population has two dominant phenotypes, $\gamma \approx 0$ and $\gamma \approx 1$, with most of the population equally divided between them when $\alpha=\beta . \quad \alpha=1=\beta \Rightarrow Q=\frac{1}{3}$, and the population is uniformly distributed across all phenotypes, that is, each phenotype has the same fraction of the total population. $\alpha=\beta>>1 \Rightarrow 0<Q<<1$ represents a unimodal symmetric distribution in which the $\gamma=0.5$ phenotype dominates, with the variance of the distribution reducing as $\alpha+\beta$ increases. $\alpha>>\beta>1 \Rightarrow 0<Q<<1$ and $\beta>>\alpha>1 \Rightarrow 0<Q<<1$ represent left-skewed and right-skewed distributions respectively, again with reducing variances as $\alpha+\beta$ increases. Stabilising and directional selection might be expected to produce $Q<<\frac{1}{3}$, while disruptive selection will produce $Q>\frac{1}{3}$.

Differentiating (11) with respect to time gives:

$$
\frac{d v}{d t}=(1-2 \hat{\gamma}) \frac{d \hat{\gamma}}{d t} Q+\hat{\gamma}(1-\hat{\gamma}) \frac{d Q}{d t} .
$$

We now substitute for the derivatives using (10):

$$
\begin{aligned}
\hat{\gamma}(1-\hat{\gamma}) \frac{d Q}{d t}= & \left.\frac{2(1-2 \hat{\gamma})}{\hat{\gamma}(1-\hat{\gamma})} \hat{\gamma}^{2}(1-\hat{\gamma})^{2} Q^{2} \frac{\partial f}{\partial \gamma}\right|_{\gamma=\hat{\gamma}}+\left.\hat{\gamma}^{2}(1-\hat{\gamma})^{2} Q^{2} \frac{\partial^{2} f}{\partial \gamma^{2}}\right|_{\gamma=\hat{\gamma}} \\
& -\left.(1-2 \hat{\gamma}) Q^{2} \hat{\gamma}(1-\hat{\gamma}) \frac{\partial f}{\partial \gamma}\right|_{\gamma=\hat{\gamma}} .
\end{aligned}
$$

Then to the accuracy of 10 :

$$
\frac{d Q}{d t}=\left.(1-2 \hat{\gamma}) Q^{2} \frac{\partial f}{\partial \gamma}\right|_{\gamma=\hat{\gamma}}+\left.\hat{\gamma}(1-\hat{\gamma}) Q^{2} \frac{\partial^{2} f}{\partial \gamma^{2}}\right|_{\gamma=\hat{\gamma}} .
$$

The $Q$ form of the variance is substituted in the equations for the population and the trait mean 10 to provide the generic eco-evolutionary model: 


$$
\begin{aligned}
\frac{d P}{d t} & =P\left(\left.f\right|_{\gamma=\hat{\gamma}}+\left.\frac{\hat{\gamma}(1-\hat{\gamma}) Q}{2} \frac{\partial^{2} f}{\partial \gamma^{2}}\right|_{\gamma=\hat{\gamma}}\right), \\
\frac{d \hat{\gamma}}{d t} & =\left.\hat{\gamma}(1-\hat{\gamma}) Q \frac{\partial f}{\partial \gamma}\right|_{\gamma=\hat{\gamma}}, \\
\frac{d Q}{d t} & =Q^{2}\left(\left.(1-2 \hat{\gamma}) \frac{\partial f}{\partial \gamma}\right|_{\gamma=\hat{\gamma}}+\left.\hat{\gamma}(1-\hat{\gamma}) \frac{\partial^{2} f}{\partial \gamma^{2}}\right|_{\gamma=\hat{\gamma}}\right) .
\end{aligned}
$$

This model is of closed form in the variables $P, \hat{\gamma}$ and $Q$, and apart from initial conditions, does not require any additional information in order to simulate

305 
When the population and its trait mean have come to equilibrium at an interior point $\left\{P^{*}, \hat{\gamma}^{*}\right\}$ (that is, when $\left.f\right|_{\left\{P^{*}, \hat{\gamma}^{*}\right\}}=0$ and $\left.\frac{\partial f}{\partial \gamma}\right|_{\left\{P^{*}, \hat{\gamma}^{*}\right\}}=0$ respectively) then $\eta=\left.\hat{\gamma}(1-\hat{\gamma}) \frac{\partial^{2} f}{\partial \gamma^{2}}\right|_{\left\{P^{*}, \hat{\gamma}^{*}\right\}}$ is a constant. Then $Q$ changes over time 330 as:

$$
Q(t)=\frac{Q_{0}}{-\eta Q_{0} t+1}
$$

where $Q_{0}$ is the initial value of $Q$. This provides a neat check on $Q$ for many eco-evolutionary systems as it may be compared with observations of how $Q$ changes in phenotype models.

Equation (16) is only useful when the populations and trait means are at, or very close to, steady state, and the trait mean is not near a bound. Then the change in $Q$ is in general driven by the local curvature of the fitness function $\left.\frac{\partial^{2} f}{\partial \gamma^{2}}\right|_{\gamma=\hat{\gamma}}$. This local curvature of the fitness function is always negative for stabilising selection, so $\eta$ is negative and $Q$ and the trait variance $v$ will decrease over time.

340 If selection is disruptive $\eta$ is positive and $Q$ and the variance can increase over time. In some cases $Q$ may diverge in finite time, and the assumption of small variance may be violated. If so, the moment based expansion (3) breaks down and the model (15) is not valid. However, the form of $d Q / d t$ suggests that $Q$ in 15 does not necessarily diverge if $\eta$ is positive, and the generic model should be capable of simulating less extreme cases of disruptive selection.

The use of $Q$ is an improvement on the direct calculation of $v$ for two reasons:

- an inverse linear relationship of the form of 16 when $\hat{\gamma}$ is near an interior equilibrium (i.e. $\hat{\gamma}(1-\hat{\gamma})$ is approximately constant) means that as $t \rightarrow \infty$ then $v \rightarrow 0$ like $t^{-1}$, and

- when $\hat{\gamma}$ approaches a boundary equilibrium, then $\hat{\gamma}(1-\hat{\gamma}) \rightarrow 0$ like $t^{-1}$, so that $v \rightarrow 0$ like $t^{-2}$ as $t \rightarrow \infty$.

The trait variance then has non-uniform behaviour as $t \rightarrow \infty$ depending on whether the trait mean $\hat{\gamma}$ is near a bound or not. The transformation $v=$ 
$\hat{\gamma}(1-\hat{\gamma}) Q$ identifies a more uniformly behaved variable $Q$.

The $Q$ differential equation gives a smooth blending of the local slope and curvature of the landscape function as the trait mean $\hat{\gamma}$ varies inside its bounded range. This substantially improves the variance calculations over evolutionary time, particularly when trait means evolve to near trait bounds.

\section{The Simulation Models}

The eco-evolutionary ecosystem we consider simulates two plant populations competing in a resource-based framework. Each population competes for its share of inorganic nutrient in order to grow, and each phenotype within each population trades off its growth against the cost of evolving defences against the allelopathic chemicals produced by its competitors. The system is modelled in its simplest form using Lotka-Volterra competition equations.

The trade-off that each phenotype in each population may make is encapsulated by a beta-distributed trade-off parameter $0 \leq \gamma \leq 1$. This parameter appears linearly in each population's growth term, and quadratically in each population's inter-population interaction term. The initial value of the tradeoff parameter is 0.5 , offering each phenotype a choice between increasing its growth rate (by increasing $\gamma$ ) and accepting a greater detrimental effect of the other population (essentially reducing its defences), or vice versa. For example, increasing growth by $50 \%$, from $\gamma=0.5$ to $\gamma=0.75$ produces a $125 \%$ increase in the detrimental effect of the competitor $\left(\gamma^{2}=0.25\right.$ to $\left.\gamma^{2}=0.5625\right)$, and vice versa. This suggests that phenotypes in larger populations may adapt to increase their growth rates at the expense of defence, while phenotypes in smaller populations may sacrifice growth to defend themselves against their more powerful competitors.

\subsection{The Population Model}

The eco-evolutionary system in population form is initially modelled using the equations (15), without the correction term on the population equation. 
The correction term is most useful in cases where the assumption of small trait variance may not be met, and the effect of including it will be examined as the final part of the analysis.

$$
\begin{aligned}
\frac{d P_{1}}{d t} & =\left.P_{1} f_{1}\left(\gamma_{1}, P_{1}, P_{2}\right)\right|_{\gamma_{1}=\hat{\gamma}_{1}}=P_{1}\left(r_{1} \hat{\gamma}_{1} N-a_{11} P_{1}-a_{12} \hat{\gamma}_{1}^{2} P_{2}\right), \\
\frac{d P_{2}}{d t} & =\left.P_{2} f_{2}\left(\gamma_{2}, P_{1}, P_{2}\right)\right|_{\gamma_{2}=\hat{\gamma}_{2}}=P_{2}\left(r_{2} \hat{\gamma}_{2} N-a_{21} \hat{\gamma}_{2}^{2} P_{1}-a_{22} P_{2}\right), \\
\frac{d \hat{\gamma}_{1}}{d t} & =\left.\hat{\gamma}_{1}\left(1-\hat{\gamma}_{1}\right) Q_{1} \frac{\partial f_{1}}{\partial \gamma_{1}}\right|_{\gamma_{1}=\hat{\gamma}_{1}}=\hat{\gamma}_{1}\left(1-\hat{\gamma}_{1}\right) Q_{1}\left(r_{1} N-2 a_{12} \hat{\gamma}_{1} P_{2}\right), \\
\frac{d \hat{\gamma}_{2}}{d t} & =\left.\hat{\gamma}_{2}\left(1-\hat{\gamma}_{2}\right) Q_{2} \frac{\partial f_{2}}{\partial \gamma_{2}}\right|_{\gamma_{2}=\hat{\gamma}_{2}}=\hat{\gamma}_{2}\left(1-\hat{\gamma}_{2}\right) Q_{2}\left(r_{2} N-2 a_{21} \hat{\gamma}_{2} P_{1}\right), \\
\frac{d Q_{1}}{d t} & =Q_{1}^{2}\left[\left.\left(1-2 \hat{\gamma}_{1}\right) \frac{\partial f_{1}}{\partial \gamma_{1}}\right|_{\gamma_{1}=\hat{\gamma}_{1}}+\left.\hat{\gamma}_{1}\left(1-\hat{\gamma}_{1}\right) \frac{\partial^{2} f_{1}}{\partial \gamma_{1}^{2}}\right|_{\gamma_{1}=\hat{\gamma}_{1}}\right] \\
& =Q_{1}^{2}\left[\left(1-2 \hat{\gamma}_{1}\right)\left(r_{1} N-2 a_{12} \hat{\gamma}_{1} P_{2}\right)+\hat{\gamma}_{1}\left(1-\hat{\gamma}_{1}\right)\left(-2 a_{12} P_{2}\right)\right], \\
\frac{d Q_{2}}{d t} & =Q_{2}^{2}\left[\left.\left(1-2 \hat{\gamma}_{2}\right) \frac{\partial f_{2}}{\partial \gamma_{2}}\right|_{\gamma_{2}=\hat{\gamma}_{2}}+\left.\hat{\gamma}_{2}\left(1-\hat{\gamma}_{2}\right) \frac{\partial^{2} f_{2}}{\partial \gamma_{2}^{2}}\right|_{\gamma_{2}=\hat{\gamma}_{2}}\right] \\
& =Q_{2}^{2}\left[\left(1-2 \hat{\gamma}_{2}\right)\left(r_{2} N-2 a_{21} \hat{\gamma}_{2} P_{1}\right)+\hat{\gamma}_{2}\left(1-\hat{\gamma}_{2}\right)\left(-2 a_{21} P_{1}\right)\right],
\end{aligned}
$$

where $N=1-P_{1}-P_{2}$. Note that all terms in equation (17) are defined for the entire trait space $0 \leq \hat{\gamma}_{1}, \hat{\gamma}_{2} \leq 1$, in contrast to equation 10 , where the factor in front of the $\left.v^{2} \frac{\partial f}{\partial \gamma}\right|_{\gamma=\hat{\gamma}}$ term in the variance equation is undefined at each bound of the trait space. An advantage of the population form of ecoevolutionary models is that they may be analytically examined for the locations and stabilities of their equilibrium points to provide synopses of their long-term dynamics. Analytic expressions for the equilibrium points of (17), and their stabilities, are provided in Appendices 88.1 and 8.2 Transcritical bifurcations of these equilibrium points are fundamental to the coexistence of competing populations in this system.

\subsection{The Phenotype Model}

The eco-evolutionary system is modelled in phenotype form, where each $P_{1}$ phenotype is identified by a subscript $i=1, \ldots, n$ and each $P_{2}$ phenotype is identified by a subscript $j=1, \ldots, m$, using the equations: 


$$
\begin{gathered}
\frac{d P_{1, i}}{d t}=P_{1, i} f_{1, i}\left(\gamma_{1, i}, P_{1}, P_{2}\right)=P_{1, i}\left(r_{1} \gamma_{1, i} N-a_{11} P_{1}-a_{12} \gamma_{1, i}^{2} P_{2}\right), \\
\frac{d P_{2, j}}{d t}=P_{2, j} f_{2, j}\left(\gamma_{2, j}, P_{1}, P_{2}\right)=P_{2, j}\left(r_{2} \gamma_{2, j} N-a_{21} \gamma_{2, j}^{2} P_{1}-a_{22} P_{2}\right),
\end{gathered}
$$

where $P_{1}=\sum_{i=1}^{n} P_{1, i}, P_{2}=\sum_{j=1}^{m} P_{2, j}$, and $N=1-P_{1}-P_{2}$. Here $i$ and $j$ identify individual phenotypes, so $P_{1, i}$ is the abundance of the $i$ th phenotype of the $P_{1}$ population, that has a trait value of $\gamma_{1, i}$, etc. The number of phenotypes $n$ in the population in this example is the same for both populations, but this is not essential. Note that this model does not include any direct interphenotype interactions, either within or without populations (i.e. $f_{1, i}$ contains only $\gamma_{1, i}$, and not $\gamma_{1, k}$ or $\gamma_{2, k}$ where $\left.k \neq i, j\right)$. The phenotypes only interact via competition for the shared inorganic nutrient resource $N$.

\subsection{Numerical Simulations}

The phenotype and population models were integrated for 1,000 time steps using a fourth-fifth order adaptive step size Runge-Kutta solver (ode45 in Matlab). The parameter values used in the integrations are shown in Table 1 The initial conditions were the same for each integration: $P_{1}=0.10=P_{2}$; $\hat{\gamma}_{1}=0.5=\hat{\gamma}_{2} ;$ and $Q_{1}=0.0244=Q_{2}$. Note each initial $Q$ was defined by the parameters of its beta distribution: $\alpha_{1}=20=\alpha_{2}$ and $\beta_{1}=20=\beta_{2}$, which were chosen to ensure that the initial beta distributions had small variances $\left(v_{1}=0.006=v_{2}\right)$. One hundred phenotypes of each population were numerically simulated. The initial trait distributions used for the phenotype simulations are shown in Figure 1 .

As noted above, we simulate four scenarios by defining four parameter sets (Table 1) that would each have different outcomes in the absence of evolution. Figure 2 shows the ecospace diagrams of the initial conditions for the average phenotypes of each scenario. The ecospaces are subsets of the population phase planes, defined by $0<P_{1}, P_{2}<1, P_{1}+P_{2}<1$, and contain all realistic solutions to the population model 17 - detailed descriptions of ecospaces are provided in Cropp and Norbury [11. Similarly, evospaces (trait spaces) are subsets of 

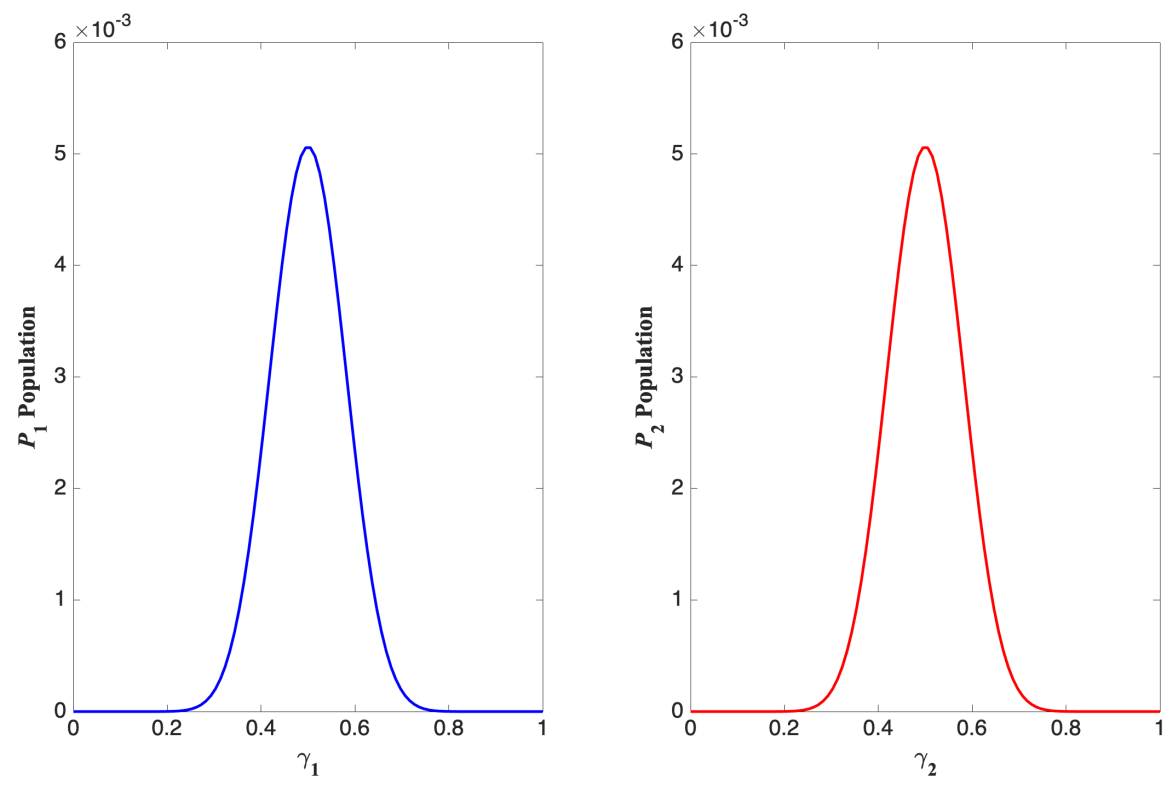

Figure 1: Initial trait distributions used for the phenotype model simulations.

Table 1: Parameter values used in simulations. Note the following scalings are applied to $\underline{\text { ensure consistency of initial conditions: } r_{1}=2 r_{1}, a_{12}=4 a_{12}, r_{2}}=2 r_{2}, a_{21}=4 a_{21}$.

\begin{tabular}{lcccccc} 
Simulation & $r_{1}$ & $a_{11}$ & $a_{12}$ & $r_{2}$ & $a_{21}$ & $a_{22}$ \\
\hline I & 1.00 & 0.35 & 0.20 & 1.10 & 0.25 & 0.35 \\
II & 1.00 & 0.75 & 0.75 & 1.10 & 0.75 & 0.75 \\
III & 1.00 & 1.50 & 1.95 & 1.01 & 2.00 & 1.50 \\
IV & 1.00 & 0.75 & 1.00 & 1.50 & 1.25 & 1.75 \\
\hline
\end{tabular}


the trait phase plane defined by $0<\gamma_{1}, \gamma_{2}<1$. Note that the properties of the ecological system shown in the ecospaces depend on the values of $\hat{\gamma}_{1}$ and $\hat{\gamma}_{2}$ used to draw the diagram, and similarly, the properties of the evolutionary system shown in the evospaces depend on the values of $P_{1}$ and $P_{2}$ used. This is because we are showing only two-dimensional subsets of the model's six-dimensional phase space.

The ecospaces in Figure 2 are drawn using $\hat{\gamma}_{1}=0.5=\hat{\gamma}_{2}$. If $Q_{1}=0=Q_{2}$ the system (17) does not evolve, and these diagrams then show the outcome of nonevolutionary scenarios of (17), which in each scenario is that the system ends up at the stable equilibrium points marked by the black dots. Non-evolutionary outcomes of the scenarios are: Scenario I - $P_{1}$ and $P_{2}$ coexist; Scenario II $P_{1}$ goes extinct and $P_{2}$ survives; Scenario III - either $P_{1}$ survives and $P_{2}$ goes extinct or vice versa, coexistence is not possible; and Scenario IV - $P_{1}$ survives and $P_{2}$ goes extinct [7, 43].

The similarities of the different model results were assessed by comparing the total population sizes, the trait means, the trait variances and estimates of $Q$ for each simulation scenario. The population model produces direct estimates of the total population sizes, the trait means, and $Q$, and then variances were calculated using equation (11). The total populations from the phenotype model were obtained by summing the abundance of each phenotype, and were then compared to the populations predicted by the population model.

The means and variances of the distributions produced by the phenotype model were calculated in the usual manner and compared to the average trait values from the population model. $Q$ values for the phenotype model were estimated from equation (11), using the mean $\hat{\gamma}$ and variance $v$ of the phenotype distributions, and compared to the directly calculated $Q$ from the population model. The trait variances for the population model were estimated from the trait means and $Q$ s produced by the model, and compared to the trait variances of the distributions produced by the phenotype model.

Note that many more simulations than are shown here were run by, for instance, varying the parameter values and/or initial conditions. Some simulations 

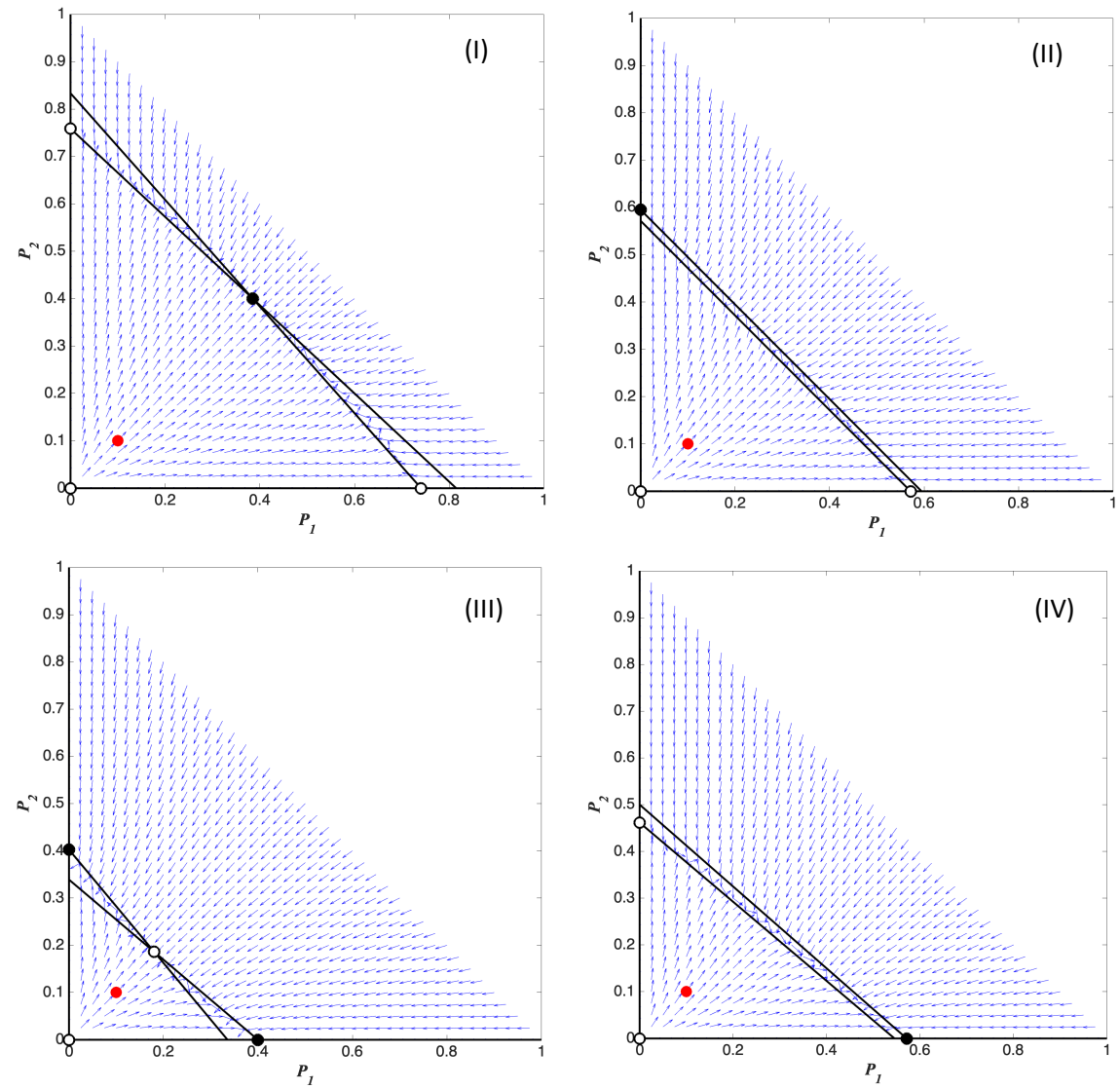

Figure 2: Initial ecospace (phase plane) diagrams for the four scenarios. Lines show the zero isoclines, where the $f_{1}$ and $f_{2}$ of the population equations are zero (note the axes are also zero isoclines), the white dots show unstable population equilibrium points, the black dots show stable population equilibrium points, and the red dots show the initial population sizes. The blue arrows show the vector field of populations, from which their dynamics at any point in the ecospace can be inferred. 
were also run out to 20,000 time steps to numerically confirm the locations and stabilities of the equilibrium points. The simulations over extended time ranges (see Appendix 8.5 for an example) confirm that the results of the two models become more similar over time in all scenarios, suggesting that the equilibrium simulations underpin the reliability of the results shown.

\section{Results and Discussion}

\subsection{Scenarios}

The first result of note from the model simulations (Figure 3 ) is the remarkably close agreement, in all scenarios, between the phenotype distributions predicted by the phenotype model and those calculated from the trait means and $Q$ values predicted by the population model. The population model distributions are calculated by deriving the trait variances from equation (11), assuming that the traits have beta distributions, and then calculating the beta distribution shape parameters $\alpha$ and $\beta$ from the trait means and variances. The trait distributions for the population model shown by the dashed lines in Figure 3 were calculated by generating beta distributions using the derived shape parameters for each trait and scaling the distribution by the final size of the population with that trait. The similarities in location, shape and magnitude of these distributions lends support to the key role of beta distributions in trait evolution.

In all four scenarios both models predict that the evolution of simple tradeoffs between growth and defence by the plant populations allows both populations to modify their traits so that the populations coexist. Some of the simulations shown in Figure 3 have not yet come to equilibrium (eg. see Figure 5. Very long integrations (up to 20,000 time steps) of the population model show that in all simulations the attracting eco-evolutionary equilibrium point $\left\{P_{1}^{*}, P_{2}^{*}, \hat{\gamma}_{1}^{*}, \hat{\gamma}_{2}^{*}\right\}$ lies on an evolutionary boundary (see Appendix 8.1), and is described by: 


$$
\left\{\begin{array}{c}
\frac{a_{22} r_{1}-a_{12} r_{2} \hat{\gamma}_{2}^{*}}{a_{11} a_{22}-a_{12} a_{21} \hat{\gamma}_{2}^{* 2}+a_{11} r_{2} \hat{\gamma}_{2}^{*}+a_{22} r_{1}-a_{21} r_{1} \hat{\gamma}_{2}^{* 2}-a_{12} r_{2} \hat{\gamma}_{2}^{*}}, \\
\frac{a_{11} r_{2} \hat{\gamma}_{2}^{*}-a_{21} r_{1} \hat{\gamma}_{2}^{* 2}}{a_{11} a_{22}-a_{12} a_{21} \hat{\gamma}_{2}^{* 2}+a_{11} r_{2} \hat{\gamma}_{2}^{*}+a_{22} r_{1}-a_{21} r_{1} \hat{\gamma}_{2}^{* 2}-a_{12} r_{2} \hat{\gamma}_{2}^{*}}, \\
1, \frac{r_{2} N^{*}}{2 a_{21} P_{1}^{*}}
\end{array}\right\},
$$

where $N^{*}=1-P_{1}^{*}-P_{2}^{*}$, in Scenarios I, III, and IV, and by:

$$
\left\{\begin{array}{c}
\frac{a_{22} r_{1} \hat{\gamma}_{1}^{*}-a_{12} r_{2} \hat{\gamma}_{1}^{* 2}}{a_{11} a_{22}-a_{12} a_{21} \hat{\gamma}_{1}^{* 2}+a_{11} r_{2}+a_{22} r_{1} \hat{\gamma}_{1}^{*}-a_{21} r_{1} \hat{\gamma}_{1}^{*}-a_{12} r_{2} \hat{\gamma}_{1}^{* 2}}, \\
\frac{a_{11} r_{2}-a_{21} r_{1} \hat{\gamma}_{1}^{*}}{a_{11} a_{22}-a_{12} a_{21} \hat{\gamma}_{1}^{* 2}+a_{11} r_{2}+a_{22} r_{1} \hat{\gamma}_{1}^{*}-a_{21} r_{1} \hat{\gamma}_{1}^{*}-a_{12} r_{2} \hat{\gamma}_{1}^{* 2}}, \\
\frac{r_{1} N^{*}}{2 a_{12} P_{2}^{*}}, 1
\end{array}\right\},
$$

in Scenario II. The locations of these eco-evolutionary equilibrium points are:

$$
\begin{array}{rll}
\text { Scenario I: } & \left\{P_{1}^{*}, P_{2}^{*}, \hat{\gamma}_{1}^{*}, \hat{\gamma}_{2}^{*}\right\}=\{0.58,0.22,1.00,0.37\}, \\
\text { Scenario II: } & \left\{P_{1}^{*}, P_{2}^{*}, \hat{\gamma}_{1}^{*}, \hat{\gamma}_{2}^{*}\right\}=\{0.07,0.63,0.17,1.00\}, \\
\text { Scenario III: } & \left\{P_{1}^{*}, P_{2}^{*}, \hat{\gamma}_{1}^{*}, \hat{\gamma}_{2}^{*}\right\}=\{0.38,0.07,1.00,0.18\}, \\
\text { Scenario IV: } & \left\{P_{1}^{*}, P_{2}^{*}, \hat{\gamma}_{1}^{*}, \hat{\gamma}_{2}^{*}\right\}=\{0.63,0.04,1.00,0.16\} .
\end{array}
$$

Details of the eco-evolutionary equilibrium points are provided in Appendix 8.1

Although the trait distributions in Figure 3 appear similar to normal distributions with small variances, equation (21) makes it clear that all simulations will asymptote to equilibrium states where at least one trait mean is one. While models that assume traits have normal distributions could be expected to produce similar initial results to equations (17), as the integrations progress and the trait means closely approach one, models that assume traits have beta distributions will produce more accurate predictions than those that assume normal distributions. Note that the $Q$ equations in the population model (17) have asymptotic equilibrium states $Q_{1}^{*}=0=Q_{2}^{*}$, as the model does not include migration or mutation that can maintain non-zero trait variances.

The population trait means in Scenario I evolve slowly, but the long integrations of the population model (Appendix 8.5) show that they eventually are 
III, and IV, in which one population is initially destined for extinction, evolve more quickly, and in each case one population adapts so that it has the larger population. The more abundant populations are primarily comprised of phenotypes with trait values near one. These phenotypes maximise their growth rate and accept the cost to their defensive abilities that this incurs.

The less abundant populations exist at comparatively low population levels, and are dominated by phenotypes with small trait values. These phenotypes sacrifice their growth rate so that they have more robust defences that protect them from the competitive influence of the more abundant population. Note those phenotypes that can successfully compete with the dominant population have been selected for. In some cases the average phenotype of the less abundant population could not compete with the average phenotype of the more abundant population, and hence would have gone extinct without adaptation, a common rescue" 20].

The ecospace diagrams of the average phenotypes at the end of the simulations (Figure 4) shows that in Scenarios II-IV the average phenotypes can now coexist, and that the end point of the simulation (the red dot) has reached ecological equilibrium as it is located at the intersection of the two zero isoclines. All the boundary equilibrium points in the ecospaces are unstable. Note this does not imply that the system has reached evolutionary equilibrium, and Figure 5 makes it clear that they have not in Scenario I.

Comparison of the time series of the phenotype and population models for 5 shows that the dynamics of the populations (upper panels) and trait means (lower panels) are almost identical. While these dynamics are mostly monotonic and quite simple, after the artefacts of the populations' initial conditions have been eliminated, the trait means appear not to have come to equilibrium, and 530

so continue to drive subtle changes in the populations, so that they also have 

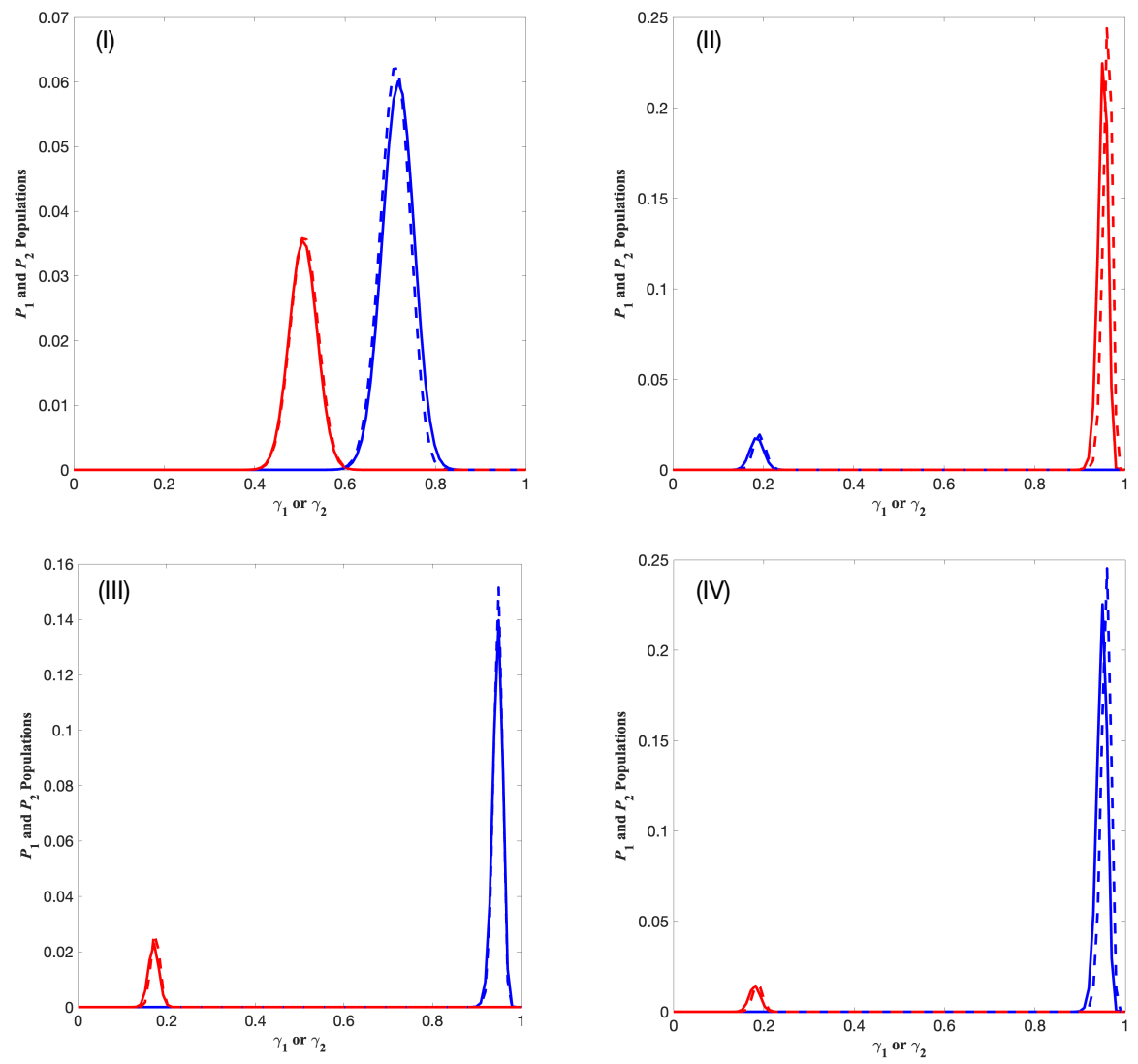

Figure 3: Phenotype distributions at the end of the integrations for the four scenarios of the phenotype model [18) (solid lines), and final distributions calculated for the four scenarios of the population model 17) (dashed lines) assuming traits have beta distributions. The trait beta distribution shape parameters $\alpha$ and $\beta$ were estimated from the final trait means and variances, where the trait variances were calculated from the final $Q$ values using equation 111. Panels show results of Scenario I (I, top left); II (II, top right); III (III, bottom left); and IV (IV, bottom right). The abundances of the $P_{1}$ phenotypes are shown in blue, and the $P_{2}$ phenotypes in red in each scenario. Note that these are near and tending to, but are not, equilibrium distributions. 

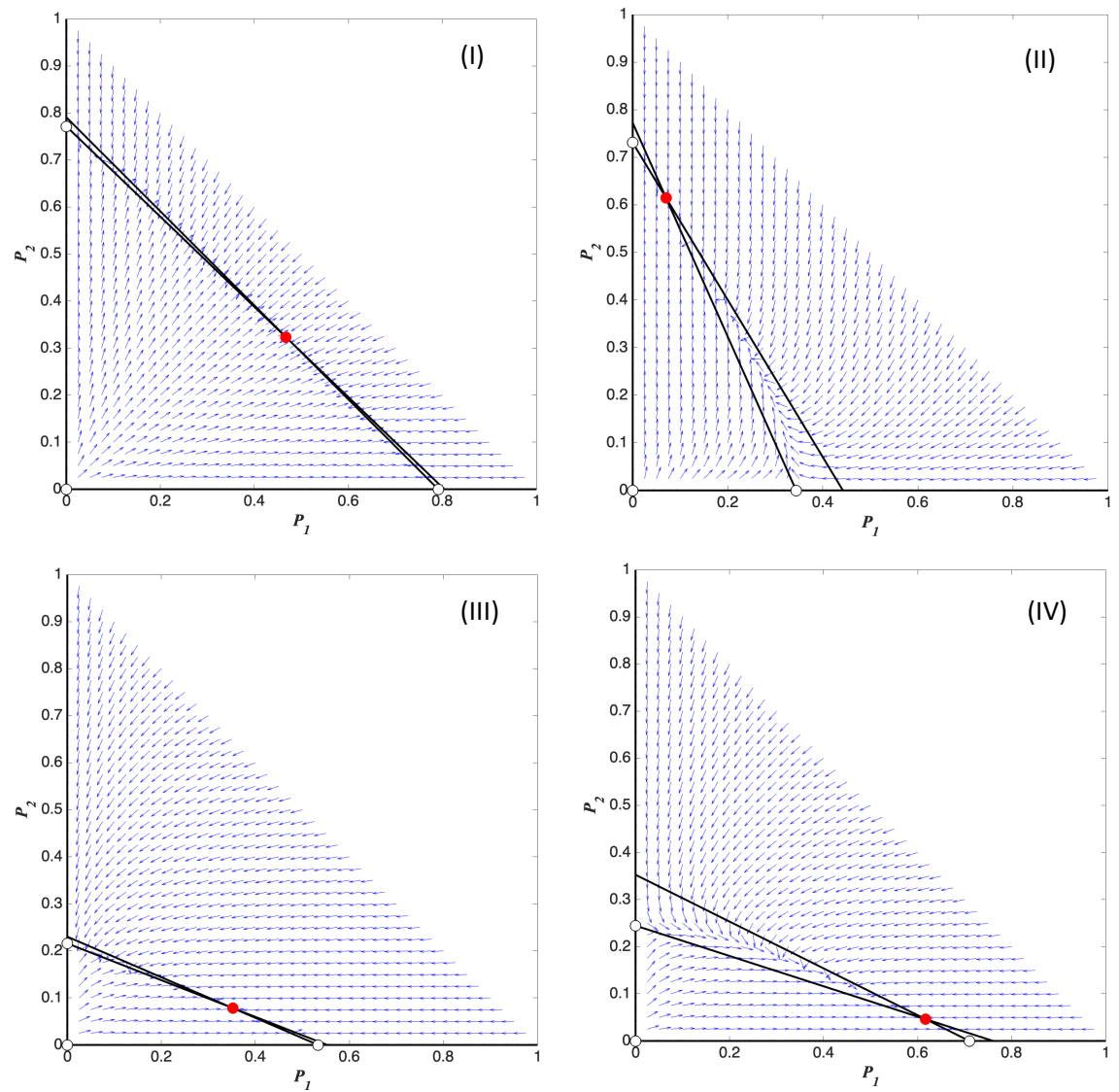

Figure 4: End of integration ecospace (phase plane) diagrams for the four scenarios of the population model (equation (17). As for Figure 2 except that red dots now show final population sizes. In all diagrams the red dot sits over a black dot indicating a stable equilibrium point. 

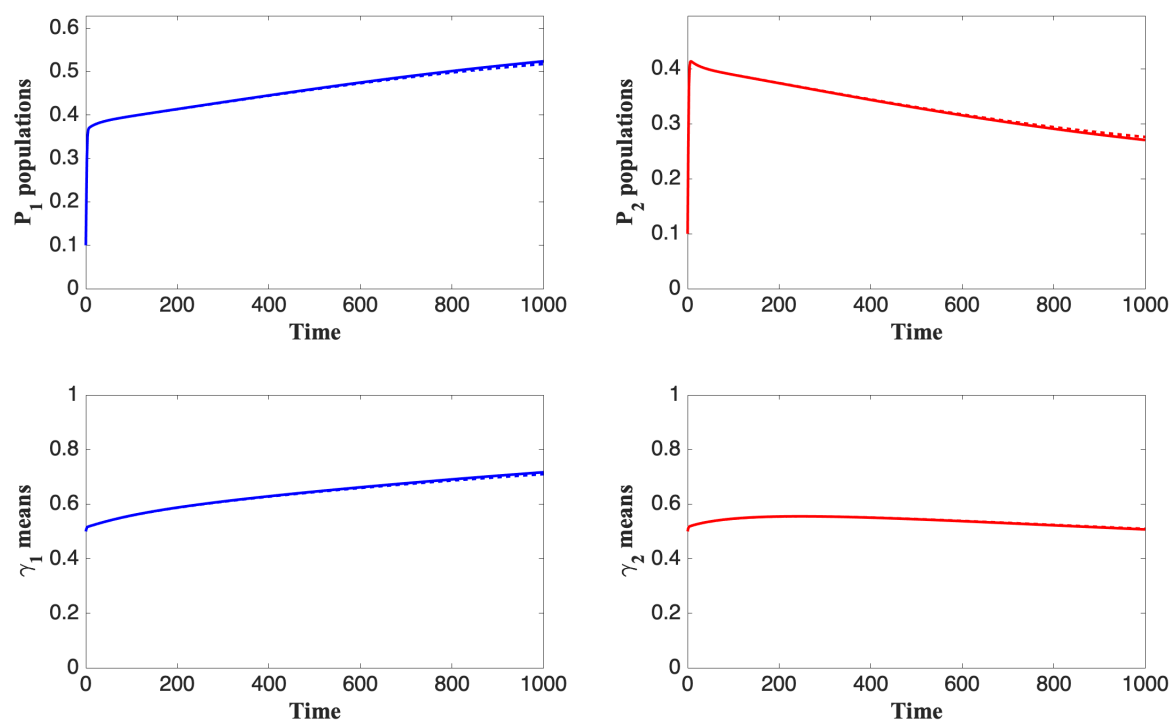

Figure 5: Scenario I: Comparisons of the time series for population sizes (top panels) and their average trait values (bottom panels) for the phenotype model (equation 18, solid lines) and the population model (equation (17), dashed lines). The left panels show $P_{1}$ attributes, and the right panels $P_{2}$ attributes. 
not yet reached equilibrium.

Analysis of the population model equations (17) reveals that the system is evolving towards the eco-evolutionary equilibrium point described by 19 and (21). The very good agreement between the population sizes and average trait values of the two models is also evident in the trait variances and $Q$ for the simulations (Figure 6), although the dynamics of these quantities through the simulations are again quite simple.
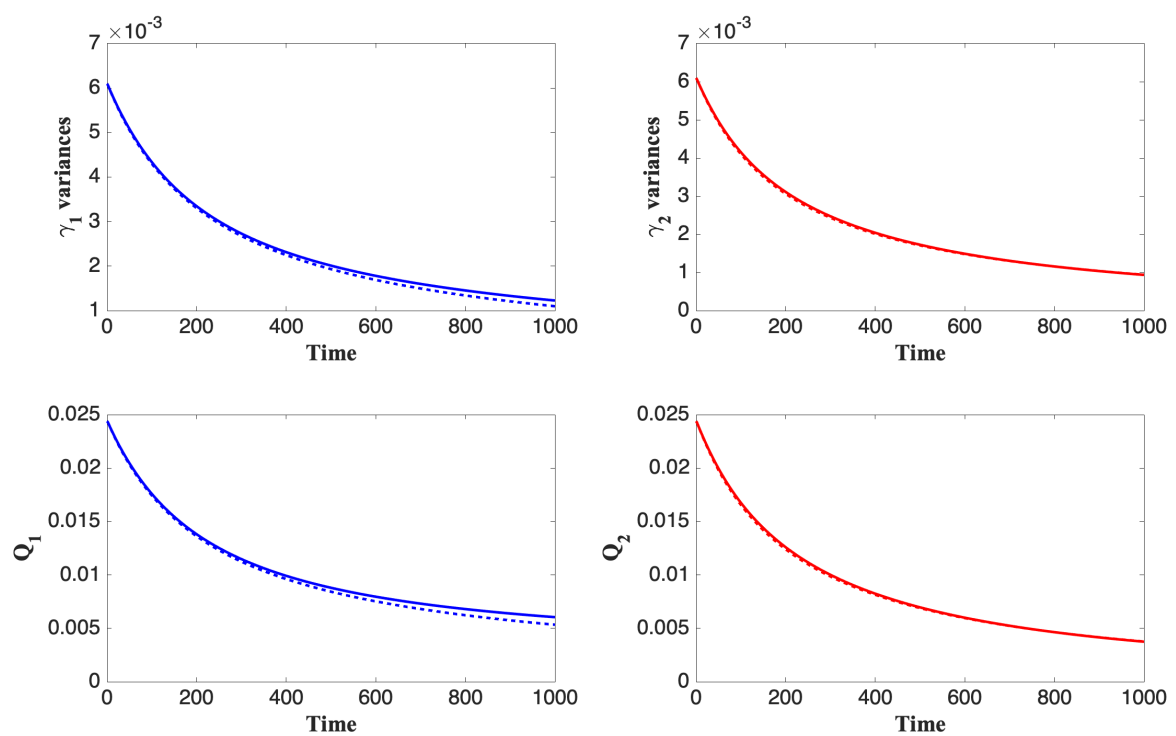

Figure 6: Scenario I: Comparisons of the time series for trait variances (top panels) and $Q$ values (bottom panels) for the phenotype model (equation 18 , solid lines) and the population model (equation (17), dashed lines).

Comparison of the model outputs for Scenario II provides more interesting results (Figures 7 and 8 . The population dynamics are much more volatile, with initial rapid increases of both populations (again, an artefact of the population initial conditions), followed by a collapse of $P_{1}$ to a very low level as $P_{2}$ dominates the system. However, $P_{1}$ adapts and slowly recovers, causing a reduction in the $P_{2}$ population as it does so. The change in trait means (Figure 

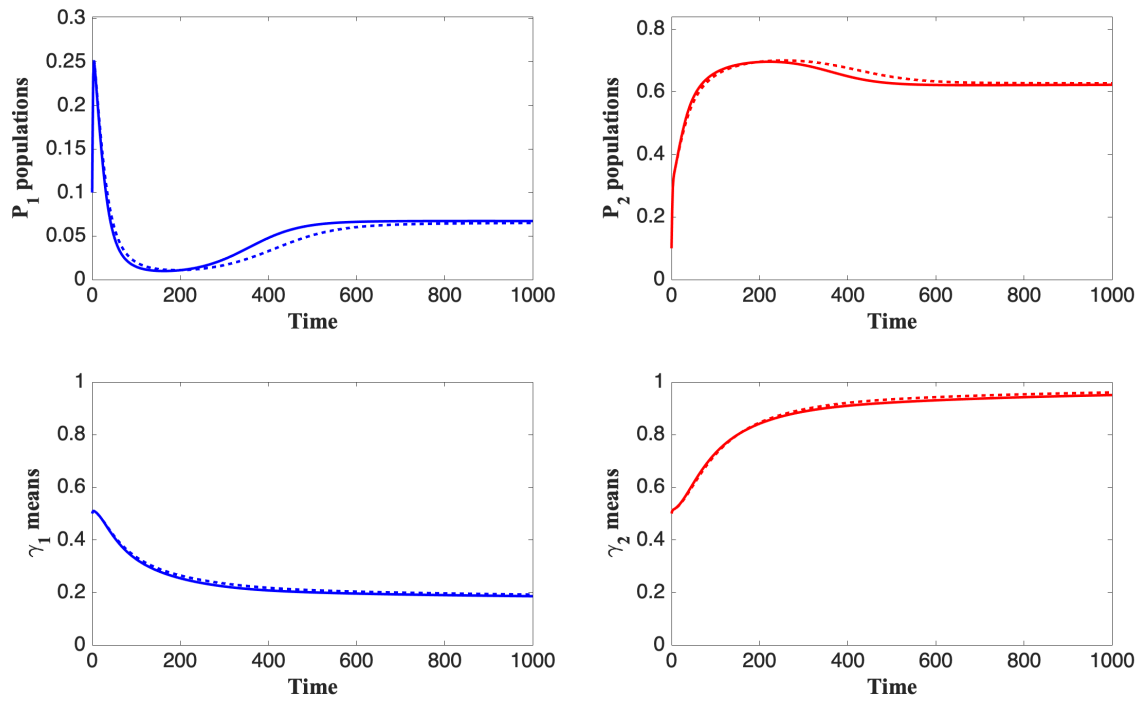

Figure 7: Scenario II: Comparisons of the time series for population sizes (top panels) and their average trait values (bottom panels) for the phenotype model (equation 18, solid lines) and the population model (equation 17 , dashed lines). 
7 lower panel) suggests that the recovery of $P_{1}$ may be attributed to its strategy 545 of improving its defences to mitigate the deleterious effects of a large $P_{2}$ population, as it reduces its trait mean, sacrificing growth for defence. Conversely, $P_{2}$ maximises its growth rate, as the detrimental effect of $P_{1}$ is minor due to its small population. These evolutionary strategies lead to coexistence of the two populations after competitively-weak phenotypes have been lost from both populations.
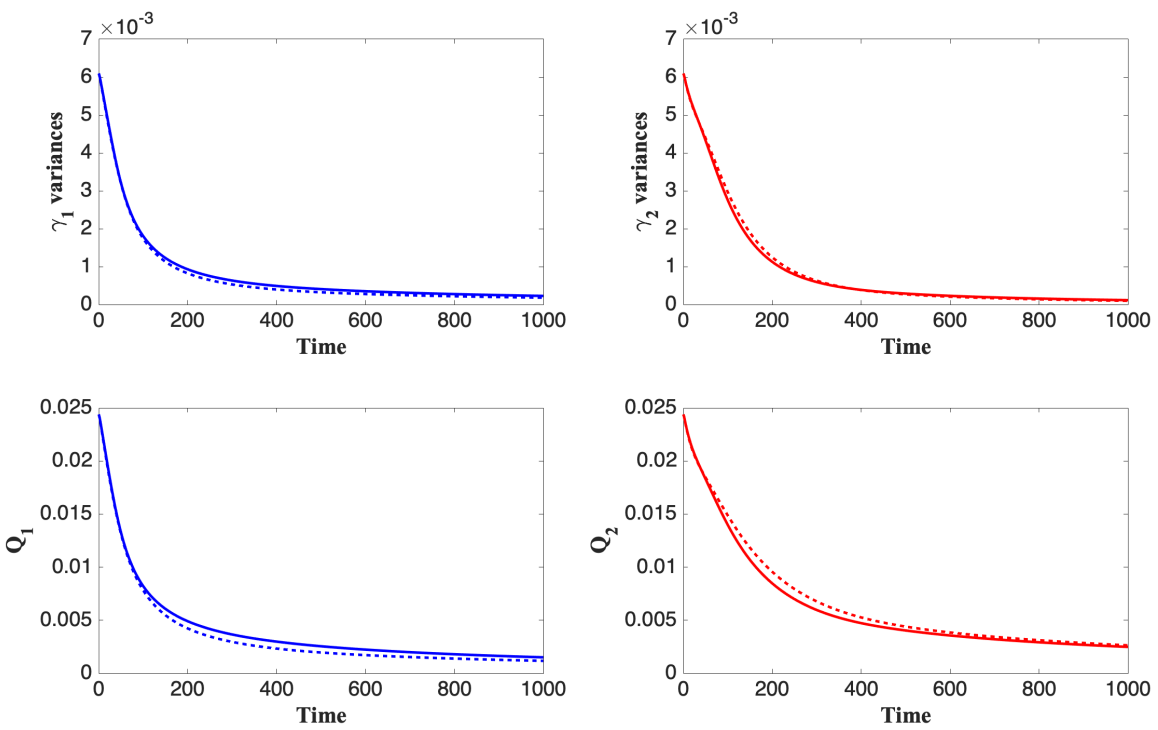

Figure 8: Scenario II: Comparisons of the time series for trait variances (top panels) and $Q$ values (bottom panels) for the phenotype model (equation [18, solid lines) and the population model (equation (17), dashed lines).

The efficacy of the population model as a surrogate of the phenotype model is again emphasised in Scenario II by the close agreement of the trait variances and $Q$ (Figure 8 ). These show excellent agreement over the entire course of the simulations. Figure 7 suggests that evolution will continue ever more slowly as $\hat{\gamma}_{2}$ slowly asymptotes to its bound, and the eco-evolutionary system to its equilibrium state (21). 

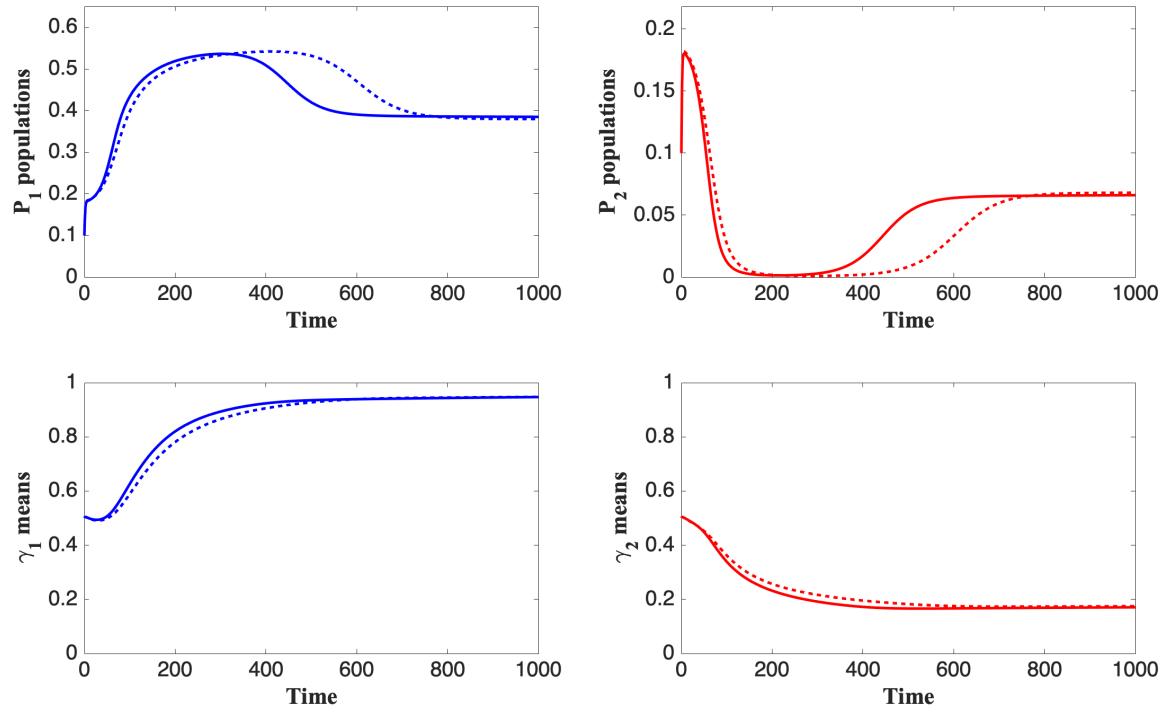

Figure 9: Scenario III: Comparisons of the time series for population sizes (top panels) and their average trait values (bottom panels) for the phenotype model (equation 18 , solid lines) and the population model (equation 17, dashed lines). 
The most significant difference between the phenotype and population model simulations in Scenario II is the subtle difference in the timing of the recovery of the $P_{1}$ population (and the commensurate slight reduction in the $P_{2}$ population). A similar difference in timing to that observed in Scenario II also occurs, and is more evident, in Scenario III (Figures 9 and 10, where the population model predicts that the recovery (of $P_{2}$ in this case) occurs significantly later than in the phenotype model.

The recovery of the $P_{2}$ population in the population model occurs some 200 time steps after the $P_{2}$ recovery in the phenotype model. However, despite the difference in timing, the populations recover to the same level, and the trait means predicted by the simulations are very similar, again suggesting a deeper congruence between the two models, as both models approach the ecoevolutionary equilibrium point $(21)$. The results of the simulations of Scenario IV are very similar to those of Scenario II, but with a much smaller difference in timing, and are presented in Appendix 8.3.

The fundamental agreement between the two models in Scenario III, which has the most substantial difference in timing of all the scenarios, is further evident in the time series of the trait variances and $Q$ (Figure 10), where only subtle differences are evident. The difference in timing of the population dynamics in Figure 9 may be attributable to fundamental differences between the phenotype and population model that only come into play when one or more populations are recovering from very small population sizes. When a population in the population model is very small, it can only increase when the mean trait value changes to give it a positive per capita growth rate $f$. However, the same population in the phenotype model, with the same total population and mean trait value, has some phenotypes that can grow, and even when the phenotype with the mean trait value cannot. This leads to some phenotypes in the phenotype model being able to recover from very small total sizes earlier than the equivalent population in the population model.

A significant advantage of the population model approach is that the system is amenable to analysis that may provide insights into the properties of the eco- 

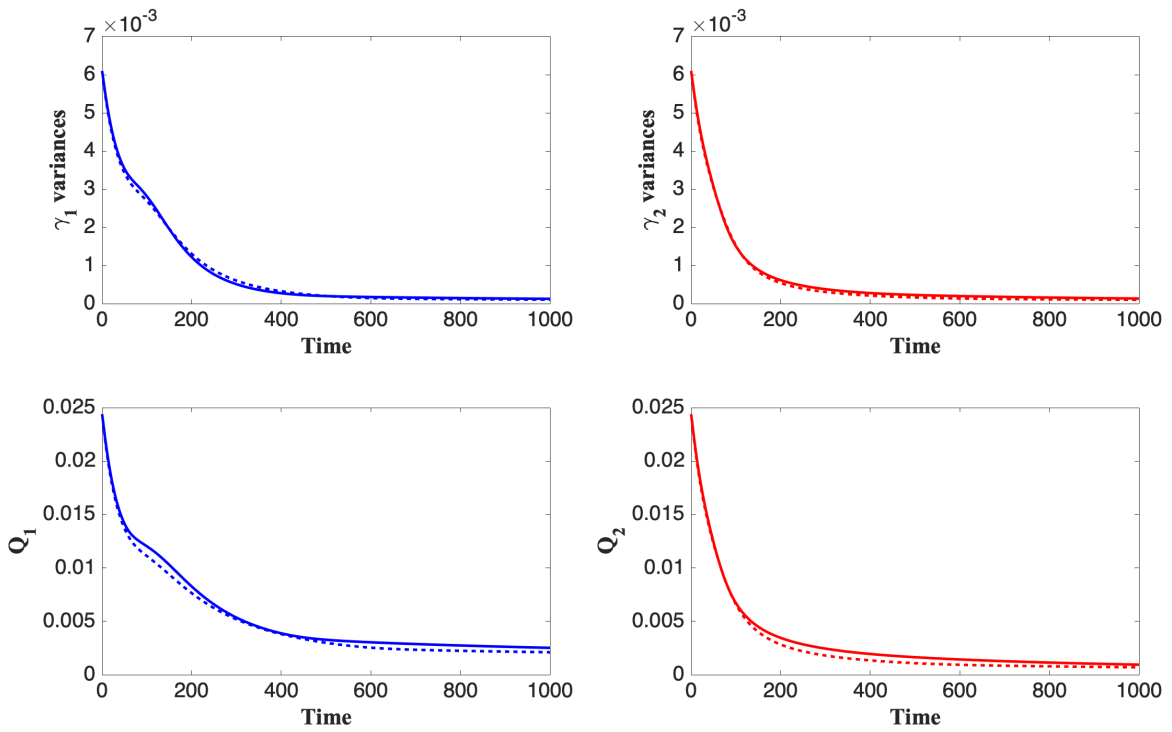

Figure 10: Scenario III: Comparisons of the time series for trait variances (top panels) and $Q$ values (bottom panels) for the phenotype model (equation $[18$, solid lines) and the population model (equation (17), dashed lines). 
evolutionary system that are not available from the phenotype model approach. Scenario III provides a good example of this, with the simple dynamics observed in Figure 9 providing little indication of the fundamental changes that occur to the properties of the model. These are described in a bifurcation analysis in Appendix 8.6 .

\subsection{Bifurcation Analysis of Scenario III}

Scenario III provides an opportunity to demonstrate the advantage of the population modelling approach over the phenotype modelling approach. Dynamical systems analysis of the population model allows us to see that profound changes to the long-term dynamical properties of the model occur due to changes in the evolutionary space while the ecology appears to be at or near equilibrium.

Figure 20 in Appendix 8.6 presents an expanded view of the dynamics of the population model populations in Scenario III, previously shown in Figure 9 The more detailed view shows that while the population abundances simulated by the model change very little between time 200 and time 300 the equilibrium state of the ecology changes significantly. The coexistence equilibrium point, which was located near $P_{1} \approx 0.3, P_{2} \approx 0.1$ for most of the initial stages of the integration, suddenly disappears off to infinity and beyond, as $P_{1}^{*} \rightarrow-\infty$ and $P_{2}^{*} \rightarrow+\infty$, around time 250. However, $P_{1}^{*}$ reappears in the ecospace from $+\infty$, and $P_{2}^{*}$ from $-\infty$ soon after, at around time 255 . This behaviour is the result of two sequential transcritical bifurcations, in which the coexistence equilibrium point first leaves the ecospace, and then re-enters.

The bifurcation analysis of Scenario III reveals that the ecosystem properties change fundamentally in the range $0.1894<\hat{\gamma}_{1} \hat{\gamma}_{2}<0.1904$. When $\hat{\gamma}_{1} \hat{\gamma}_{2}>$ 0.1904 the ecosystem has an unstable coexistence equilibrium point and two stable boundary equilibrium points, so that either $P_{1}$ or $P_{2}$ will dominate and send the other population to extinction. When $\hat{\gamma}_{1} \hat{\gamma}_{2}<0.1894$ the ecosystem has a stable coexistence equilibrium point and two unstable boundary equilibrium 
points, so that the two populations will coexist. The system comes to ecoevolutionary equilibrium with $\hat{\gamma}_{1}^{*} \hat{\gamma}_{2}^{*} \approx 0.1749$.

The bifurcation analysis reveals a key insight into eco-evolutionary dynamics, hat an eco-evolutionary system that is ostensibly at ecological equilibrium may in fact be far from eco-evolutionary equilibrium, even though as Figure 9 shows, both the ecology and the trait means are changing slowly. Further, despite the very slow change in the trait means, the properties of the ecology may change dramatically as a result of very subtle changes in the trait means.

\subsection{Correction Terms}

Finally, we examine the effect of increasing the initial trait variance on the performance of the population model. The simulations shown in Figures 5 - 10 (and Figures 14 and 15 in Appendix 8.3) commence with initial beta distributions that have $\alpha=20=\beta$ which implies an initial trait mean of 0.5 and an initial trait variance of 0.006. The simulation results shown in Figures 11 and 12 commence with an initial trait mean of 0.5 and a larger initial trait variance of 0.012 (i.e $\alpha=10=\beta$ ).

Comparison of Figures 9 and 11 shows that the change in initial trait variance has caused a slight change to the timing of the population dynamics for both the

${ }_{35}$ population and phenotype models, but the overall dynamics remain similar (see Appendix 8.4. The differences in timing of the population dynamics appears to be related to the exponential recoveries of the phenotypes and populations from small values.

When $P_{2}$ becomes small in the population model simulations, the timing of 640 its recovery depends upon the "smallness", compared to the variance, of the whole population, which recovers only when the mean trait value allows it to grow. However, in the phenotype model, when the phenotype with the mean trait value is unable to grow, some phenotypes are able to grow due to the variance in phenotype traits. These phenotypes will begin their exponential recovery from small values before the population model. Populations with large trait variances will have greater numbers of phenotypes with trait values that 
allow them to grow than the equivalent phenotype model simulation with small variance, and therefore those phenotypes will recover more rapidly than both the population models and the phenotype models with small variances. This phenomenon is explained further in 8.4 of the Appendix.

The increased initial trait variance has led to bigger differences between the population model estimate of the population dynamics compared to the phenotype model (blue and red lines respectively in Figure 11 upper panels). The corrected population model (shown by the black lines) provides a much better estimate of the population dynamics for the initial dynamics, and the transition through the evolutionary bottleneck, although all estimates of the final population states are similar. The inclusion of the correction terms:

$$
\begin{aligned}
& \left.\frac{1}{2} \hat{\gamma}_{1}\left(1-\hat{\gamma_{1}}\right) Q_{1} \frac{\partial^{2} f_{1}}{\partial \gamma_{1}^{2}}\right|_{\gamma_{1}=\hat{\gamma}_{1}}=\frac{1}{2} \hat{\gamma}_{1}\left(1-\hat{\gamma}_{1}\right) Q_{1}\left(-2 a_{12} P_{2}\right), \\
& \left.\frac{1}{2} \hat{\gamma}_{2}\left(1-\hat{\gamma_{2}}\right) Q_{2} \frac{\partial^{2} f_{2}}{\partial \gamma_{2}^{2}}\right|_{\gamma_{2}=\hat{\gamma}_{2}}=\frac{1}{2} \hat{\gamma}_{2}\left(1-\hat{\gamma}_{2}\right) Q_{2}\left(-2 a_{21} P_{1}\right),
\end{aligned}
$$

to the $P_{1}$ and $P_{2}$ equations respectively in $(17)$ substantially improves the agreement between the population estimates of the phenotype and population models if the assumption of small variance is relaxed. Including the population correction terms does not, however, eliminate the time lags evident in Scenario III, and to lesser extents in Scenarios II and IV.

Figure 11 reveals that increasing the initial trait variance has a much lesser effect on the estimates of the trait mean values, although the uncorrected model produces slightly, but noticeably, worse estimates. Figure 12 reveals that there is even less difference between the trait variances and $Q$ values predicted by the corrected and uncorrected population models, and that both are very similar to the predictions of the phenotype model.

These comparisons emphasise that the population model is an approximation 670 to the phenotype model, and that the verisimilitude of the approximation depends on the number of terms taken in the expansions that the approximations are based on. The population model described in equation (17) assumes that 

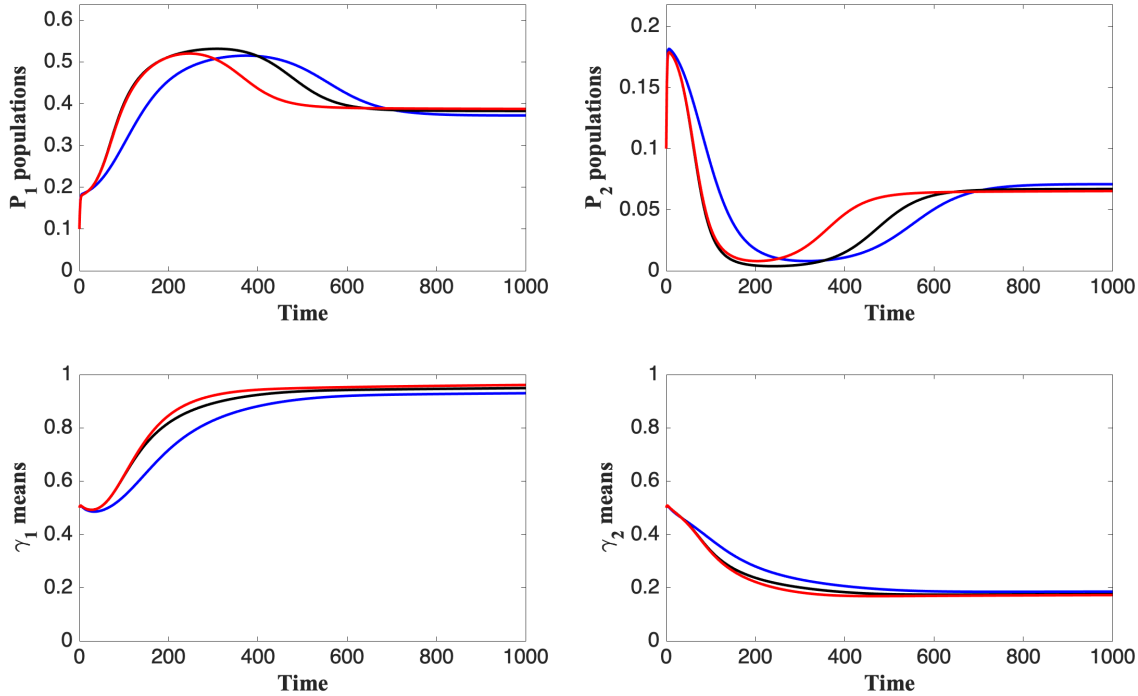

Figure 11: Scenario III with increased initial trait variance: Comparisons of the time series for population sizes (top panels) and their average trait values (bottom panels) for the phenotype model (equation 18, red lines), the population model (equation 17), blue lines), and the population model with the correction term included in the population equation (as shown in equation 15 , black lines). 

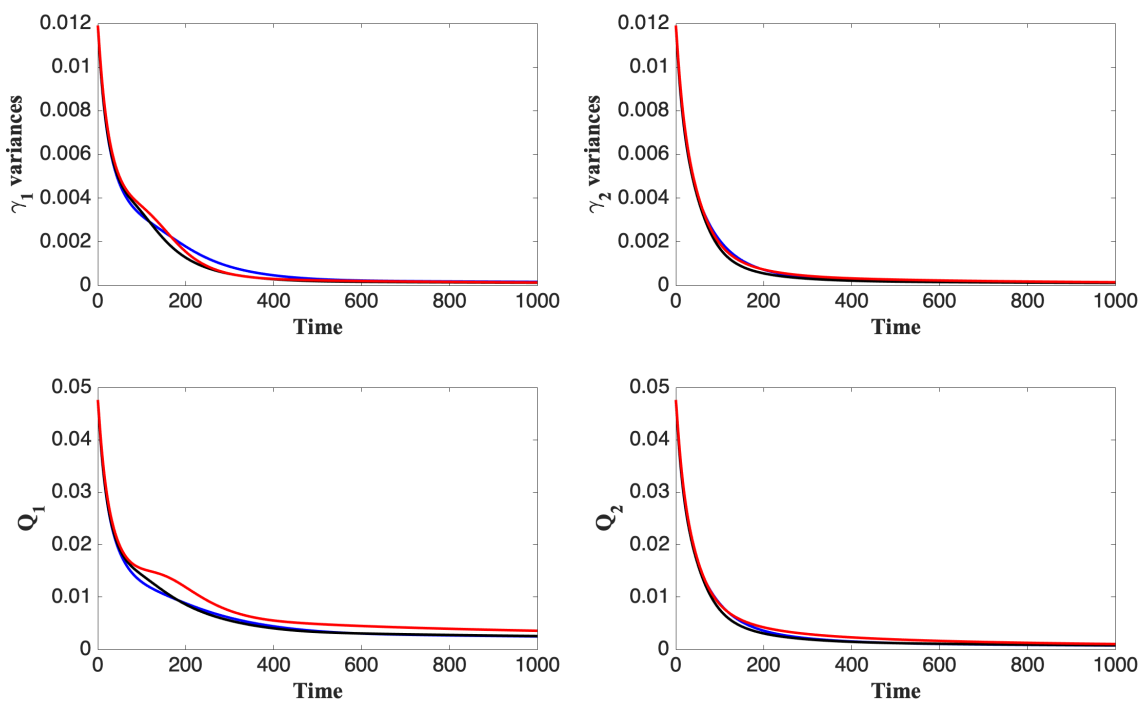

Figure 12: Scenario III with increased initial trait variance: Comparisons of the time series for trait variances (top panels) and $Q$ (bottom panels) for the phenotype model (equation [18, red lines), the population model (equation (17), blue lines), and the population model with the correction term included in the population equation (as shown in equation 15), black lines). 
the traits are beta-distributed with "small" variance. The simulations shown in Figures 11 and 12 confirm that when the assumption of small variance is relaxed simulations may maintain accuracy if additional terms are included in the equations. Additional terms may also be required to resolve evolutionary processes for simulations over very long time scales.

\section{Conclusions}

The primary conclusion drawn from this research is that the population model using $Q$ is an excellent, simpler representation of the detailed phenotype model (a surrogate for "reality"). The example modelling of competing populations also provides some insights into the trade-off of growth and defence characteristics. We note that the general model equations 15 are not restricted to such simple examples, but may be applied to a spectrum of cases including stabilising, directional and disruptive selection, examples of which will be provided in future work.

The influence of evolutionary processes to allow coexistence of populations that would otherwise be mutually exclusive is not of itself a new idea, for example [1, 2, 154, 34, 17, 21], but there are interesting aspects to our results. We deal with the comparison of the modelling approaches first.

The most important result of this work is the excellent agreement between the predictions of the phenotype and population models. The phenotype model simulates the outcome of competition within and between populations with hundreds of phenotypes, each with a trait representing a slightly different trade-off, while the population model simulates the total populations, the average trait (trade-off) of each population, and how the level of phenotypic differentiation changes within each population. The agreement is evident across all direct and indirectly calculated population metrics: the total population sizes $P_{1}$ and $P_{2}$, the average trait values $\hat{\gamma}_{1}$ and $\hat{\gamma}_{2}$, the trait variances $v_{1}$ and $v_{2}$, and the phenotypic differentiations $Q_{1}$ and $Q_{2}$. It is worth noting in this context that the phenotype model predicts only the abundance of each phenotype in each popu- 
lation - the summary statistics are calculated from these distributions without any assumptions.

The very close agreement between the results produced by the population model, which requires that traits always have beta distributions, and the phenotype model, that has no restrictions on the functional form that the trait distribution may take, suggest that the key assumptions that underpin the population model are reasonable. These assumptions include that:

- some trait parameter values are naturally bounded;

- some trait parameter values may be effectively represented by beta distributions;

- the fitness of a phenotype or the average fitness of a population may be effectively represented by its life function $f$; and

- our $Q$ may be used as an effective tool to simulate the change in variance of a population's phenotypes that occurs due to increased differentiation within the population that results from a loss of less fit phenotypes.

The results also support some interesting conclusions, in particular that:

- the eco-evolutionary dynamics of ecosystems with populations that have small variance in their phenotypes may be accurately simulated by simple population models with few "correction" terms, such as equations (17);

- eco-evolutionary models that assume beta-distributed traits, and are created using $Q$ are of closed form, and do not require other information to effectively simulate eco-evolutionary systems, an advantage over previous moment closure approaches that assume beta-distributed traits [27];

725

- the population model equations synthesise the actual phenotype interactions exceedingly accurately provided the model's basic assumption of small phenotypic variance is respected, and quite accurately if it is not; and 
- the accuracy of simulations of the eco-evolutionary dynamics of populations with larger phenotypic variances may be improved by including additional terms in the equations.

We note that including additional terms in the equations, while improving the before- and after-bottleneck agreement between the phenotype and population models, does not eliminate the difference in the timing of the recovery of the population from its bottleneck.

A key difference between the phenotype and population models is that the population model makes assumptions about the fitness of the average phenotype, how the average fitness of the population changes, and the distribution of phenotypes (traits) within each population. The phenotype model includes none of these assumptions and effectively provides a "reality" to check the population model results against.

The ecological implications of the modelling results are also interesting. The four scenarios presented have simple, consistent outcomes in non-evolutionary models. In Scenario I, both populations coexist; in Scenarios II and IV either $P_{2}$ or $P_{1}$ respectively survives and the competing population goes extinct according to $R^{*}$ theory [53]; and in Scenario III either $P_{1}$ or $P_{2}$ (but not both) will survive and the other will go extinct depending on the initial sizes of the populations. In each scenario, the larger population (after the initial conditions have been washed out) adopts a strategy of maximising its growth rate without trade-off 750 (its $\hat{\gamma} \nearrow 1$ ) as its competitor has only a small detrimental effect on it. Its competitor is forced to defend itself and must trade-off growth against defense, so its $\hat{\gamma} \searrow \hat{\gamma}^{*}>0$. Adding evolutionary processes to the models changes the outcomes for Scenarios II-IV, so that evolution may facilitate coexistence and diversity among competitors [1, although this may not occur in cases of convergent evolution [16].

Of particular interest in the simulations we present here is the behaviour of $Q$. The simulation results of the phenotype model imply that while the variance of the phenotype distribution in the population may initially reduce 
rapidly, and $Q$ commensurately initially reduces rapidly, this reduction slows.

The relative coarseness of the phenotype model (only 100 distinct phenotypes in each population are simulated) suggests that we could expect that the single most fit phenotype would soon dominate the population, and all other phenotypes would go extinct. In a long simulation this would occur (unless multiple phenotypes had identical fitnesses), but the phenotype simulations suggest that very long simulation times are required to achieve this.

The population model reproduces this behaviour accurately, with $Q$ tending to, but not reaching zero, and so maintaining small, but non-zero, variances in the phenotype distributions. This is consistent with equation 16 which reveals that $Q$ tends to zero slowly as time increases. After the populations and trait means have closely approached their equilibrium values, $Q$ reduces as $1 /\left[-\left.\hat{\gamma}(1-\hat{\gamma}) \frac{\partial^{2} f}{\partial \gamma^{2}}\right|_{\gamma=\hat{\gamma}} t\right]$.

\section{Closing Remarks}

This work has introduced a key new concept to eco-evolutionary modelling, the use of $Q$ as a measure of phenotypic differentiation within a population. This, with the recognition that many traits are constrained in the values they may realistically take, and the subsequent assumption that beta distributions are an appropriate descriptor of phenotype abundances within populations, produces an eco-evolutionary population model with two important attributes.

First, the moment-based expansions used to develop the model are explicit, and closed differently to previous authors that have assumed beta distributions; no additional information is needed to solve the model. Second, the simulation of $Q$ rather than the variance eliminates the singularities in the variance equation that occur at the trait boundaries, allowing trait means to evolve to their bounds as and when the phenotype model indicates this should happen.

The universality of the new eco-evolutionary modelling framework (15) rests on the validity of its major assumptions relating to evolutionary processes, that:

- the traits have beta distributions, 
- the trait distributions have small variances,

- the per capita growth rate is a suitable measure of fitness,

790

795

- the analogy to the phenotypic differentiation, that we call $Q$, is a useful means of simulating how the shape of the beta-distributions of traits changes as the population evolves.

The close agreement between simulations using the population model scenarios that include these assumptions, and the equivalent phenotype model scenarios that do not, suggests that the assumptions are reasonable and the modelling framework is robust.

The assumption of beta, rather than Gaussian, distributed traits does not appear particularly restrictive due to the ability of the beta distribution to approximate many other distributions. A key difference is that beta distributions are bounded, as the values of many traits will be, and in cases where the bounds are not defined by the ecological properties of the populations, the setting of appropriate bounds is required.

The assumption of small variance appears to preclude the application of this approach to disruptive selection, that may cause a population to split into subpopulations discriminated by different trait value modes and consequent large population trait variance. However, the model may be modified to cope with larger variances and slower processes by including additional correction terms. We expect that this model has the potential to accurately simulate a spectrum of eco-evolutionary scenarios, and provide analytic insights into those scenarios that are not available through other model formulations.

\section{Acknowledgements}

The authors wish to thank two anonymous reviewers for their considered and constructive comments on the original manuscript. The review process has led to a much improved and more readable paper. 
[1] Abrams, P., 1986. Character displacement and niche shift analyzed using consumer-resource models of competition. Theor Pop Biol 29, 107-160.

[2] Abrams, P., 2012. The eco-evolutionary responses of a generalist consumer to resource competition. Evolution 66, 3130-3143.

[3] Abrams, P., Harada, Y., Matsuda, H., 1993. On the relationship between quantitative genetic and ess models. Evolution 47, 982-985.

[4] Abrams, P., Matsuda, H., 2004. Consequences of behavioral dynamics for the population dynamics of predator-prey systems with switching. Popul Ecol 46, 13-25.

[5] Balding, D., Nichols, R., 1995. A method for quantifying differentiation between populations at multi-allelic loci and its implications for investigating identity and paternity. Genetica 96, 3-12.

[6] Brommer, J., 2011. Whither $p_{S T}$ ? the approximation of $q_{S T}$ by $p_{S T}$ in evolutionary and conservation biology. Journal of Evolutionary Biology 24, 1160-1168.

[7] Case, T.J., 2000. An illustrated guide to theoretical ecology. Oxford University Press, New York.

[8] Champagnat, N., 2006. A microscopic interpretation for adaptive dynamics trait substitution sequence models. Stochastic Processes and their Applications 116, 1127-1160.

[9] Cortez, M., 2016. How the magnitude of prey genetic variation alters predator-prey eco-evolutionary dynamics. Am Nat 188, 329-341.

[10] Coutinho, R., Klauschies, T., Gaedke, U., 2016. Bimodal trait distributions with large variances question the reliability of trait-based aggregate models. Theoretical Ecology 9, 389-408. 
[11] Cropp, R., Norbury, J., 2015. Population interactions in ecology: A rulebased approach to modeling ecosystems in a mass-conserving framework. SIAM Rev 57, 437-465.

[12] Cropp, R., Norbury, J., 2019a. An eco-evolutionary system with naturally bounded traits. Theoretical Ecology, doi:10.1007/s12080-019-0407-6.

[13] Cropp, R., Norbury, J., 2019b. Obligate mutualism in a resource-based framework. SIAM Review 61, 596-622.

[14] Cropp, R., Norbury, J., 2021. Modelling the evolution of naturally-bounded traits in a population. Theoretical Ecology https://doi.org/10.1007/s12080020-00495-2, 1-14.

[15] Doebeli, M., 2011. Adaptive Diversification and Speciation as Pattern Formation in Partial Differential Equation Models. Princeton University Press. pp. 217-261. URL: http://www.jstor.org/stable/j.ctt7rgw4. 12 .

[16] Edwards, K., Kremer, C., Miller, E., Osmond, M., Litchman, E., Klausmeier, C., 2018. Evolutionarily stable communities: a framework for understanding the role of trait evolution in the maintenance of diversity. Ecology Letters 21, 1853-1868.

[17] Farahpour, F., Saeedghalati, M., Brauer, V., Hoffmann, D., 2018. Trade-off shapes diversity in eco-evolutionary dynamics. eLIFE 7, e36273.

[18] Geritz, S., Kisdi, E., Meszena, G., Metz, J., 1998. Evolutionarily singular strategies and the adaptive growth and branching of the evolutionary tree. Evolutionary Ecology 12, 35-57.

[19] Geritz, S., Metz, J., Kisdi, E., Meszena, G., 1997. Dynamics of adaptation and evolutionary branching. Physical Review Letters 78, 2024-2027.

[20] Gomulkiewicz, R., Holt, R., 1995. When does evolution by natural selection prevent extinction? Evolution 49, 201-207. 
[21] Govaert, L., Fronhofer, E., Lion, S., Eizaguirre, C., Bonte, D., Egas, M., Hendry, A., De Brito Martins, A., Melian, C., Raeymaekers, J., Ratikainen, I., Saether, B., Schweitzer, J., Matthews, B., 2019. Eco-evolutionary feedbacks -theoretical models and perspectives. Functional Ecology 33, 13-30.

[22] Hastings, A., Powell, T., 1991. Chaos in a three-species food chain. Ecology $72,896-903$.

[23] Iwasa, Y., Pomiankowski, A., 1991. The evolution of costly mate preferences II. the "handicap" principle. Evolution 45, 1431-1442.

[24] Johnson, D., 1997. The triangular distribution as a proxy for the beta distribution in risk analysis. The Statistician 46, 387-398.

[25] Kempes, C., Koehl, M., West, G., 2019. The scales that limit: The physical boundaries of evolution. Frontiers in Ecology and Evolution 7, doi:10.3389/fevo.2019.00242.

[26] Klais, R., Norros, V., Lehtinen, S., Tamminen, T., Olli, K., 2016. Community assembly and drivers of phytoplankton functional structure. Functional Ecology 31, 760-767.

[27] Klauschies, T., Coutinho, R., Gaedke, U., 2018. A beta distribution-based moment closure enhances the reliability of trait-based aggregate models for natural populations and communities. Ecological Modelling 381, 46-77.

[28] Klausmeier, C., Kremer, C., Koffel, T., 2020. Trait-based ecological and eco-evolutionary theory. Oxford University Press. chapter 11. pp. 161-194.

[29] Kolmogorov, A., 1936. Sulla teoria di volterra della lotka per l'esisttenza. Giornale dell Instituto Italiano Degli Attuari 7, 74-80.

[30] Lande, R., 1976. Natural selection and random genetic drift in phenotype evolution. Evolution 30, 314-334.

[31] Lande, R., 1979. Quantitative genetic analysis of multivariate evolution, applied to brain: body size allometry. Evolution 33, 402-416. 
[32] Law, A., Kelton, W., 1982. Simulation Modelling and Analysis. New York: McGraw-Hill.

[33] Leinonen, T., O'Hara, R., Cano, J., Merila, J., 2008. Comparative studies of quantitative trait and neutral marker divergence: a meta-analysis. Journal of Evolutionary Biology 21, 1-17.

[34] Levine, J., Bascompte, J., Adler, P., Allesina, S., 2017. Beyond pairwise mechanisms of species coexistence in complex communities. Nature 546, $56-64$.

[35] Lewontin, R., Krakauer, J., 1973. Distribution of gene frequency as a test of the theory of the selective neutrality of polymorphisms. Genetics 74 , 175-195.

[36] Lion, S., 2018. Theoretical approaches in evolutionary ecology: environmental feedback as a unifying perspective. The American Naturalist 191, $21-44$.

[37] Lotka, A., 1925. Elements of Physical Biology. Wilkins and Kilkins, Baltimore, USA.

[38] May, R., 1976. Simple mathematical models with very complicated dynamics. Nature 261, 459-467.

[39] Meszéna, G., Gyllenberg, M., Pásztor, L., Metz, J.A., 2006. Competitive exclusion and limiting similarity: a unified theory. Theoretical Population Biology 69, 68-87.

[40] Metz, J., 2012. Adaptive Dynamics. California University Press. pp. 7-17.

[41] Norberg, J., Swaney, D., Dushoff, J., Lin, J., Casagrandi, R., Levin, S., 2001. Phenotypic diversity and ecosystem functioning in changing environments: A theoretical framework. PNAS 98, 11376-11381.

[42] Parmesan, C., 2006. Ecological and evolutionary responses to recent climate change. Annu. Rev. Ecol. Evol. Syst. 37, 637-669. 
[43] Pastor, J., 2017. Ecosystem ecology and evolutionary biology, a new frontier for experiments and models. Ecosystems 20, 245-252.

[44] Pease, C., Lande, R., Bull, J., 1989. A model of population growth, dispersal and evolution in a changing environment. Ecology 70, 1657-1664.

[45] Pham-Gia, T., Duong, Q., 1989. The generalised beta- and f-distributions in statistical modelling. Mathematical and Computational Modelling 12, $1613-1625$.

[46] Ronce, O., Kirkpatrick, M., 2001. When sources become sinks: migrational meltdown in heterogeneous habitats. Evolution 55, 1520-1531.

[47] Roughgarden, J., 1972. Evolution of niche width. The American Naturalist $106,683-718$.

[48] Schmid, M., Guillame, F., 2017. The role of phenotypic plasticity on population differentiation. Heredity 119, 214-225.

[49] Senthilnathan, A., Gavrilets, S., 2021. Ecological consequences of intraspecific variation in coevolutionary systems. The American Naturalist 197, $1-17$.

[50] Slatkin, M., 1979. Frequency-and density-dependent selection on a quantitative character. Genetics 93, 755-771.

940 [51] Spitze, K., 1993. Population structure in Daphnia obtusa: Quantitative genetic and allozymic variation. Genetics 135, 367-374.

[52] Tataru, P., Simonsen, M., Bataillon, T., Hobolth, A., 2017. Statistical inference in the wright-fisher model using allele frequency data. Systems Biology 66, e30-e46.

945 [53] Tilman, D., 1977. Resource competition between planktonic algae: an experimental and theoretical approach. Ecology 58, 338-348. 
[54] Vasseur, D., Amarasekare, P., Rudolf, V., Levine, J., $2011 . \quad$ Ecoevolutionary dynamics enable coexistence via neighbor-dependent selection. The American Naturalist 178, E96-E109.

[55] Vitousek, P., Matson, P., 2012. Nutrient cycling and biogeochemistry, in: Levin, S. (Ed.), The Princeton Guide to Ecology. Princeton University Press, Princeton New Jersey, pp. 330-339.

[56] Volterra, V., 1926. Variazione e fluttuazini del numero d'individuals in specie animali conviventi. Mem. Accad. Nazionale Linceri (Ser. IV) 2, 31113.

[57] Ward, B., Collins, S., Dutkiewicz, S., Gibbs, S., Bown, P., Ridgwell, A., Sauterey, B., Wilson, J., Oschlies, A., 2019. Considering the role of adaptive evolution in models of the ocean and climate system. Journal of Advances in Modeling Earth Systems, https://doi.org/10.1029/2018MS001452.

[58] Weir, B., Cockerham, C., 1984. Estimating $F$-statistics for the analysis of population structure. Evolution 38, 1358-1370.

[59] Whitlock, M., 2008. Evolutionary inference from $Q_{S T}$. Molecular Ecology $17,1885-1896$.

[60] Wright, S., 1950. Genetical structure of populations. Nature 166, 247-249.

[61] Wright, S., 1951. The genetical structure of populations. Ann. Eugenics $15,323-354$.

[62] Yamamichi, M., Klauschies, T., Miner, B., van Velsen, E., 2019. Modelling inducible defences in predator-prey interactions: assumptions and dynamical consequences of three distinct approaches. Ecology Letters 22, 390-404.

\section{Appendix}




\subsection{Equilibrium Points}

The eco-evolutionary population model (17) has degenerate equilibrium points at $Q_{i}^{*}=0$; on linear analysis these points are neutrally stable in the $Q_{i}$ direcrium points where either $P_{1}$ or $P_{2}$ is extinct (either $P_{1}^{*}=0$ or $P_{2}^{*}=0$ ). For $P_{1}^{*} \neq 0, P_{1}^{*}=r_{1} \hat{\gamma}_{1}^{*} /\left(r_{1} \hat{\gamma}_{1}^{*}+a_{11}\right)$, so that $N^{*}, \hat{\gamma}_{1}^{*}>0$ which forces $\hat{\gamma}_{1}^{*}=1$. Similar arguments apply for $P_{2}^{*}$, and then the six boundary equilibrium points are:

$$
\begin{aligned}
& \left\{\frac{r_{1}}{r_{1}+a_{11}}, 0,1,0\right\},\left\{\frac{r_{1}}{r_{1}+a_{11}}, 0,1,1\right\},\left\{\frac{r_{1}}{r_{1}+a_{11}}, 0,1, \frac{a_{11} r_{2}}{2 a_{21} r_{1}}\right\}, \\
& \left\{0, \frac{r_{2}}{r_{2}+a_{22}}, 0,1\right\},\left\{0, \frac{r_{2}}{r_{2}+a_{22}}, 1,1\right\},\left\{0, \frac{r_{2}}{r_{2}+a_{22}}, \frac{a_{22} r_{1}}{2 a_{12} r_{2}}, 1\right\} .
\end{aligned}
$$

990

Then four coexistence equilibrium points of the model (17), where $P_{1}^{*}, P_{2}^{*}>$ 0 , are internal to (i.e. not on the boundaries of) the ecospace. Three of these lie on the boundaries of the evolutionary space:

0,1 give four cases each. The ecology origin is where both populations are extinct, $P_{1}^{*}=0=P_{2}^{*}$, and then $N^{*}=1$, giving four possibilities for $\left\{P_{1}^{*}, P_{2}^{*}, \hat{\gamma}_{1}^{*}, \hat{\gamma}_{2}^{*}\right\}$ :

$$
\{0,0,0,0\}, \quad\{0,0,0,1\},\{0,0,1,0\},\{0,0,1,1\}
$$

these equilibrium points are always unstable.

A further six possibilities lie at the edge of the ecospace, boundary equilib- 


$$
\begin{aligned}
& \left\{\begin{array}{c}
\frac{a_{22} r_{1}-a_{12} r_{2}}{a_{11} a_{22}-a_{12} a_{21}+a_{11} r_{2}+a_{22} r_{1}-a_{21} r_{1}-a_{12} r_{2}}, \\
\frac{a_{11} r_{2}-a_{21} r_{1}}{a_{11} a_{22}-a_{12} a_{21}+a_{11} r_{2}+a_{22} r_{1}-a_{21} r_{1}-a_{12} r_{2}}, \\
1,1
\end{array}\right\}, \\
& \left\{\begin{array}{c}
\frac{a_{22} r_{1}-a_{12} r_{2} \hat{\gamma}_{2}^{*}}{a_{11} a_{22}-a_{12} a_{21} \hat{\gamma}_{2}^{* 2}+a_{11} r_{2} \hat{\gamma}_{2}^{*}+a_{22} r_{1}-a_{21} r_{1} \hat{\gamma}_{2}^{* 2}-a_{12} r_{2} \hat{\gamma}_{2}^{*}}, \\
\frac{a_{11} r_{2} \hat{\gamma}_{2}^{*}-a_{21} r_{1} \hat{\gamma}_{2}^{* 2}}{a_{11} a_{22}-a_{12} a_{21} \hat{\gamma}_{2}^{* 2}+a_{11} r_{2} \hat{\gamma}_{2}^{*}+a_{22} r_{1}-a_{21} r_{1} \hat{\gamma}_{2}^{* 2}-a_{12} r_{2} \hat{\gamma}_{2}^{*}}, \\
1, \frac{r_{2} N^{*}}{2 a_{21} P_{1}^{*}}
\end{array}\right\}, \\
& \left\{\begin{array}{c}
\frac{a_{22} r_{1} \hat{\gamma}_{1}^{*}-a_{12} r_{2} \hat{\gamma}_{1}^{* 2}}{a_{11} a_{22}-a_{12} a_{21} \hat{\gamma}_{1}^{* 2}+a_{11} r_{2}+a_{22} r_{1} \hat{\gamma}_{1}^{*}-a_{21} r_{1} \hat{\gamma}_{1}^{*}-a_{12} r_{2} \hat{\gamma}_{1}^{* 2}}, \\
\frac{a_{11} r_{2}-a_{21} r_{1} \hat{\gamma}_{1}^{*}}{a_{11} a_{22}-a_{12} a_{21} \hat{\gamma}_{1}^{* 2}+a_{11} r_{2}+a_{22} r_{1} \hat{\gamma}_{1}^{*}-a_{21} r_{1} \hat{\gamma}_{1}^{*}-a_{12} r_{2} \hat{\gamma}_{1}^{* 2}}, \\
\frac{r_{1} N^{*}}{2 a_{12} P_{2}^{*}}, 1
\end{array}\right\},
\end{aligned}
$$

and one is an internal equilibrium point of the eco-evolutionary space:

$$
\left\{\begin{array}{c}
\frac{a_{22} r_{1} \hat{\gamma}_{1}^{*}-a_{12} r_{2} \hat{\gamma}_{1}^{* 2} \hat{\gamma}_{2}^{*}}{a_{11} a_{22}-a_{12} a_{21} \hat{\gamma}_{1}^{* 2} \hat{\gamma}_{2}^{* 2}+a_{11} r_{2} \hat{\gamma}_{2}^{*}+a_{22} r_{1} \hat{\gamma}_{1}^{*}-a_{21} r_{1} \hat{\gamma}_{1}^{*} \hat{\gamma}_{2}^{* 2}-a_{12} r_{2} \hat{\gamma}_{1}^{* 2} \hat{\gamma}_{2}^{*}}, \\
\frac{a_{11} r_{2} \hat{\gamma}_{2}^{*}-a_{21} r_{1} \hat{\gamma}_{1}^{*} \hat{\gamma}_{2}^{* 2}}{a_{11} a_{22}-a_{12} a_{21} \hat{\gamma}_{1}^{* 2} \hat{\gamma}_{2}^{* 2}+a_{11} r_{2} \hat{\gamma}_{2}^{*}+a_{22} r_{1} \hat{\gamma}_{1}^{*}-a_{21} r_{1} \hat{\gamma}_{1}^{*} \hat{\gamma}_{2}^{* 2}-a_{12} r_{2} \hat{\gamma}_{1}^{* 2} \hat{\gamma}_{2}^{*}}, \\
\frac{r_{1} N^{*}}{2 a_{12} P_{2}^{*}}, \frac{r_{2} N^{*}}{2 a_{21} P_{1}^{*}}
\end{array}\right\}
$$

where $N^{*}=1-P_{1}^{*}-P_{2}^{*}$.

995

\subsection{Equilibrium Point Stability}

We examine four scenarios that each begin with a different stability of the internal and/or boundary ecological equilibrium points $\left\{P_{1}^{*}, P_{2}^{*}\right\}$. The arrangements of the zero isoclines, the vector fields and the stabilities of the equilibrium points are shown in Figure 2. Scenario I has all the boundary points unstable and the internal point stable, so both populations can initially coexist. Scenarios II and IV are initially in $R^{*}$ outcomes, where the population that leaves the least resource for the other drives the other population to extinction: in Scenario II $P_{2}$ dominates, and in Scenario IV $P_{1}$ dominates. Scenario III begins in a state where both the population boundary equilibrium points are stable, and the internal point is unstable, so either population might dominate (depending upon the initial population sizes) but the two populations cannot coexist. 
The local stability of the equilibrium points in the trait space, henceforth called the evospace, assuming the ecology is at equilibrium, are determined by the eigenvalues:

$$
\begin{aligned}
& \lambda_{\hat{\gamma}_{1}}=Q_{1}\left[\left(1-2 \hat{\gamma}_{1}^{*}\right)\left(r_{1} N^{*}-2 a_{12} \hat{\gamma}_{1}^{*} P_{2}^{*}\right)-2 \hat{\gamma}_{1}^{*}\left(1-\hat{\gamma}_{1}^{*}\right) a_{12} P_{2}^{*}\right], \\
& \lambda_{\hat{\gamma}_{2}}=Q_{2}\left[\left(1-2 \hat{\gamma}_{2}^{*}\right)\left(r_{2} N^{*}-2 a_{21} \hat{\gamma}_{2}^{*} P_{1}^{*}\right)-2 \hat{\gamma}_{2}^{*}\left(1-\hat{\gamma}_{2}^{*}\right) a_{21} P_{1}^{*}\right],
\end{aligned}
$$

where $N^{*}=1-P_{1}^{*}-P_{2}^{*}$. Note that the small variance assumption $\left\{Q_{1}, Q_{2}>0\right\}$ means that the eigenvalues in equation $(28)$ are generally non-zero.

The four possible outcomes of the evolutionary processes in the population model (17) are shown in Figure 13 . Note that in all outcomes the equilibrium point $\hat{\gamma}_{1}^{*}=0=\hat{\gamma}_{2}^{*}$ is unstable. Panel (a) shows that when the equilibrium population sizes of $P_{1}^{*}$ and $P_{2}^{*}$ are sufficiently large compared to the remaining inorganic nutrient $N^{*}$ the interior equilibrium point, where $0<\hat{\gamma}_{1}^{*}, \hat{\gamma}_{2}^{*}<1$, exists within the evospace and is stable. All the remaining evolutionary equilibrium points on the boundaries of the evospace are unstable.

Panel (b) of Figure 13 shows the influence of $P_{2}^{*}$ falling to a low population level, here 0.05. The interior equilibrium point has left the evospace through a transcritical bifurcation with the $\left\{\hat{\gamma}_{1}^{*}=1, \hat{\gamma}_{2}^{*}=0.35\right\}$ boundary equilibrium point, which has now become stable. This is the result for Scenarios I, III, and IV. Should the $P_{1}^{*}$ equilibrium population fall to small levels instead the reverse happens and we get panel (c), where the interior equilibrium point leaves 225 the evospace through a transcritical bifurcation with the $\left\{\hat{\gamma}_{1}^{*}=0.15, \hat{\gamma}_{2}^{*}=1\right\}$ boundary equilibrium point, which now is stable. This is the result of Scenario II. Should the equilibrium size of both populations be small, both the internal zero isoclines lie outside the evospace, and the $\left\{\hat{\gamma}_{1}^{*}=1, \hat{\gamma}_{2}^{*}=1\right\}$ boundary equilibrium point becomes stable, usually after a sequence of two transcritical bifurcations. 

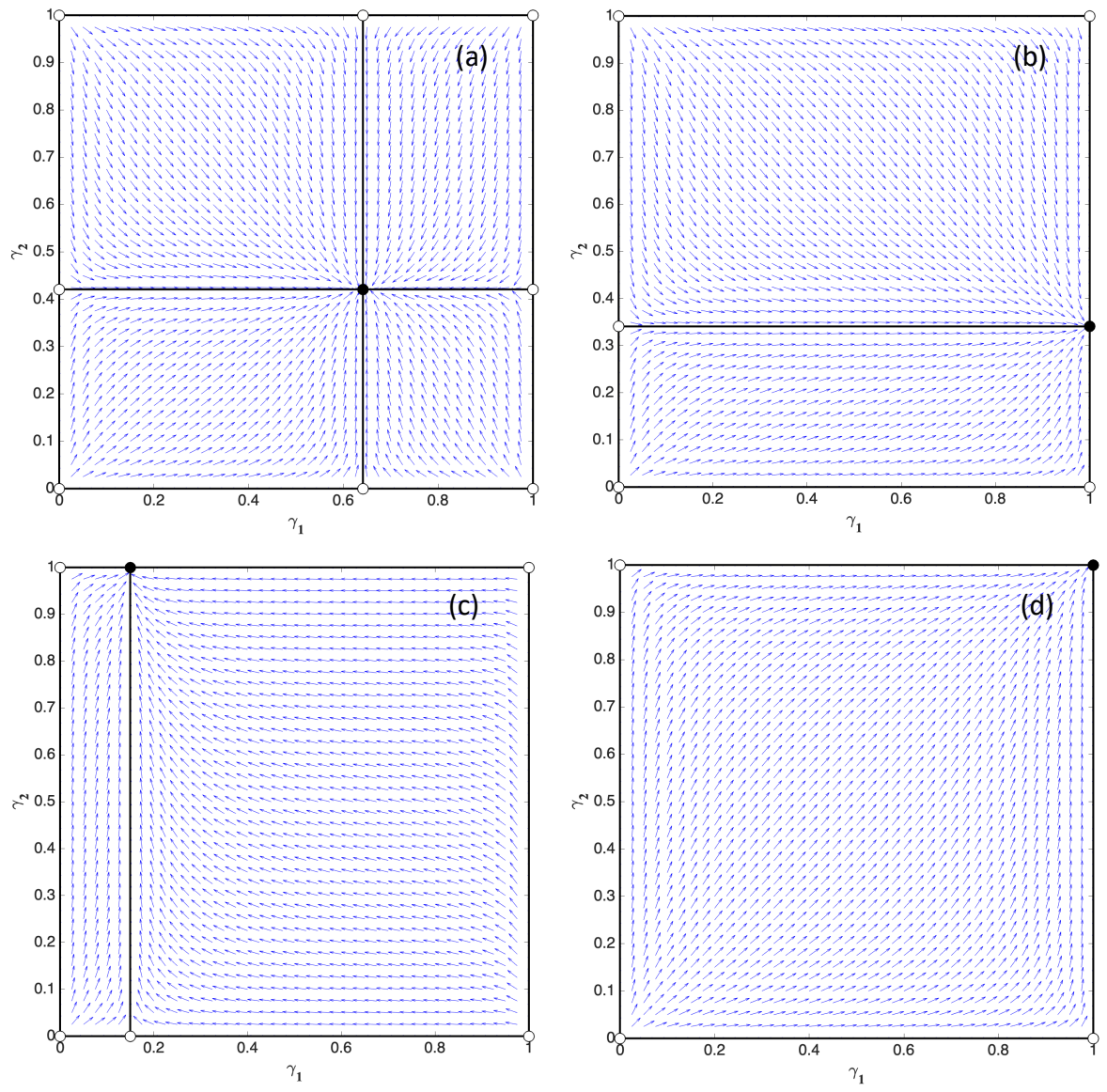

Figure 13: Evospace (phase plane) diagrams showing possible evolutionary outcomes. Lines show the zero isoclines, where the righthand sides of the evolution equations 17 are zero (note the axes are also zero isoclines), the white dots show unstable equilibrium points, and the black dots show stable equilibrium points. The blue arrows show the vector field of mean trait values, from which their dynamics at any point in the evospace can be inferred. Parameter values for Scenario III were used to draw the diagrams with: (a) $P_{1}=0.3$ and $P_{2}=0.2$; (b) $P_{1}=0.4$ and $P_{2}=0.05$; (c) $P_{1}=0.05$ and $P_{2}=0.6$; (d) $P_{1}=0.05$ and $P_{2}=0.1$. 


\subsection{Scenario IV}

The results of Scenario IV are not substantively different to those of Scenario II, with the phenotype and population models producing near identical results, and the populations again adapting to facilitate coexistence. Comparison of the three scenarios in which one population closely approaches zero at some stage of the integration (Scenarios II, III, and IV; Figures 7, 9, 14, and 16 shows that the magnitude of the time lag between the recovery of the small population in the phenotype and population models is correlated with the smallness, compared to the variance, and the duration of smallness, of the recovering population.
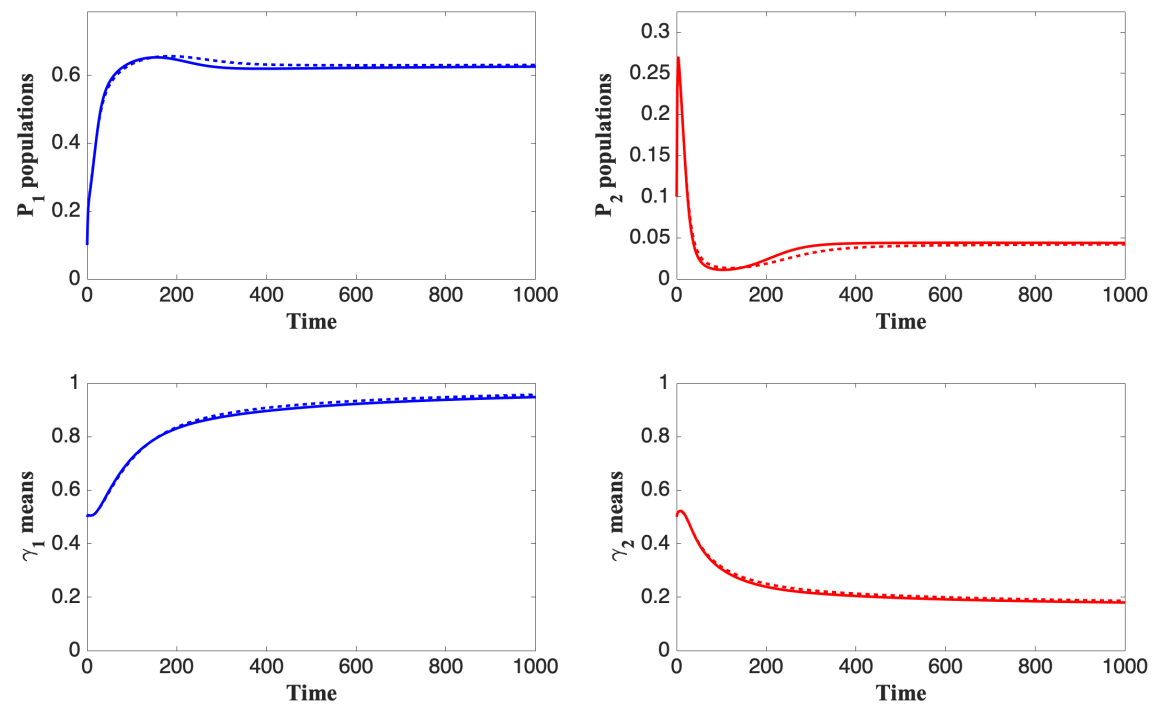

Figure 14: Scenario IV: Comparisons of the time series for population sizes (top panels) and their average trait values (bottom panels) for the phenotype model (equation [18, solid lines) and the population model (equation 17 , dashed lines).

\subsection{Effect of Initial Variance on Population Dynamics}

Additional simulations of the phenotype model (equation (18), the population model (equation (17)), and the corrected population model (equation 

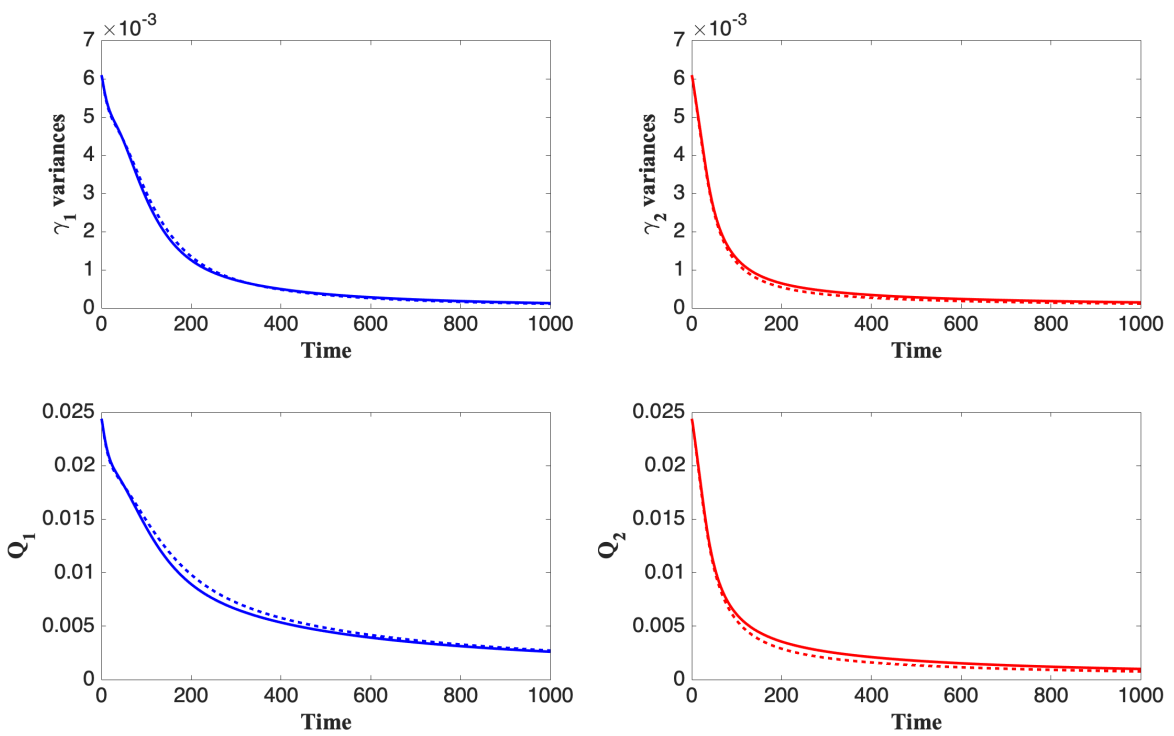

Figure 15: Scenario IV: Comparisons of the time series for trait variances (top panels) and $Q$ values (bottom panels) for the phenotype model (equation [18, solid lines) and the population model (equation (17), dashed lines). 
(17) with the extra term specified in equation (15)), were run for small initial variances $\left(\alpha_{1}=40=\alpha_{2}\right.$ and $\left.\beta_{1}=40=\beta_{2}\right)$ and large initial variances $\left(\alpha_{1}=10=\alpha_{2}\right.$ and $\left.\beta_{1}=10=\beta_{2}\right)$ to investigate the effect of initial trait variances on the model dynamics (Figures 16 and 17 ).
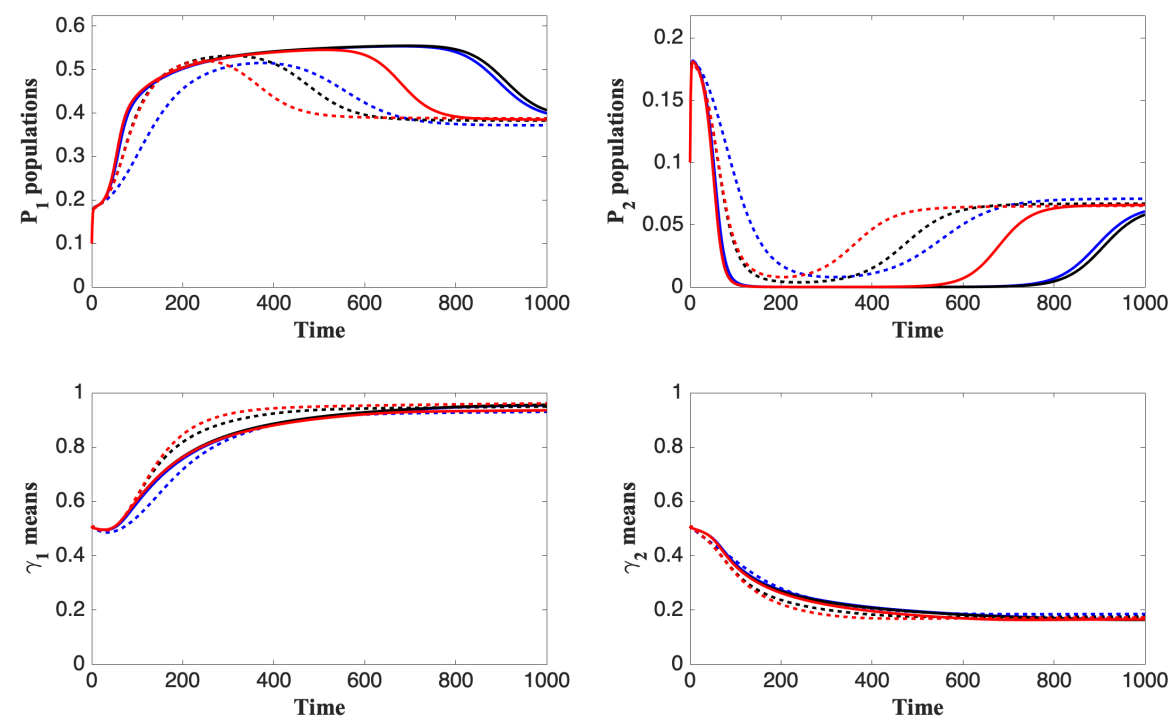

Figure 16: Comparisons of population dynamics (upper panels) and trait means (lower panels) for small variance (solid lines) and large variance (dotted lines) simulations of the phenotype model (equation 18, red lines), the population model (equation (17), blue lines), and the corrected population model (equation 17 with the extra term specified in equation 15, black lines). The small variance simulations are initiated with $\alpha_{1}=40=\alpha_{2}$ and $\beta_{1}=40=\beta_{2}$; the large variance simulations are initiated with $\alpha_{1}=10=\alpha_{2}$ and $\beta_{1}=10=\beta_{2}$.

These simulations revealed that the population dynamics timing differences between the phenotype and population models are more pronounced in the small variance simulations than in the large variance simulations. However, while the correction term added to the population equations did not noticeably improve the small variance simulations, it did reduce the difference between the phenotype and population models in the large variance simulations. Further, all models showed that the population dynamics of the large variance simulations 
were significantly different to those of the small variance simulations for all
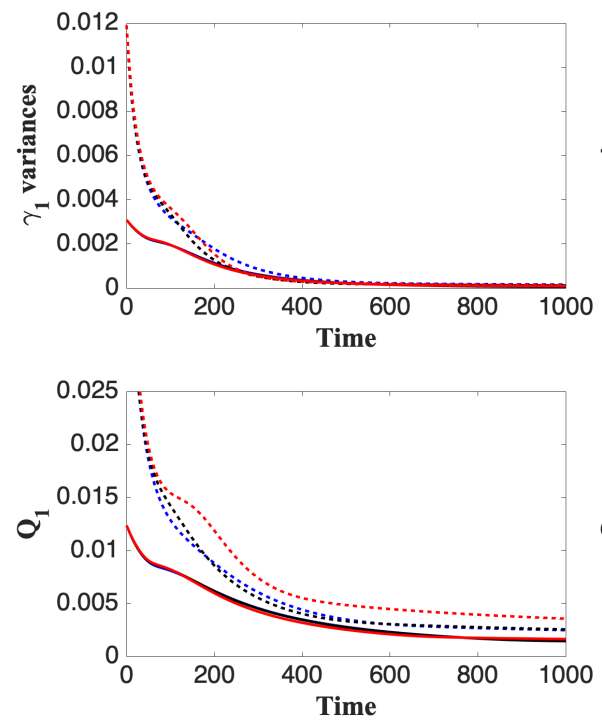
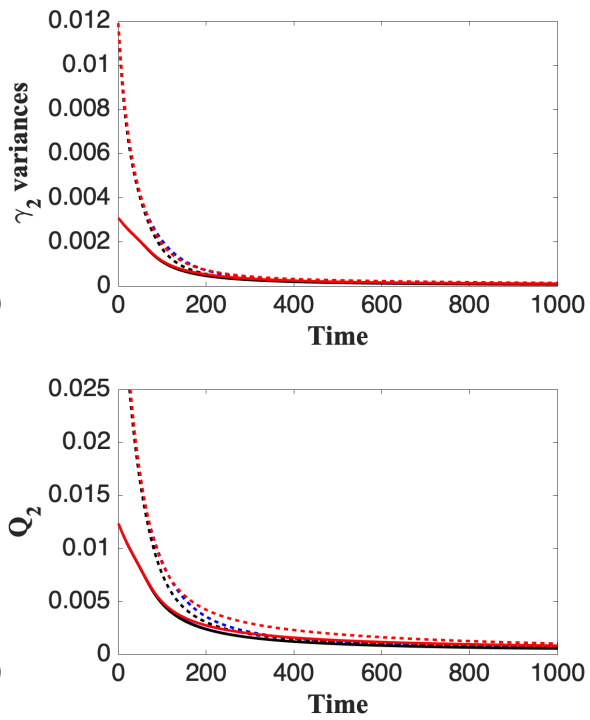

Figure 17: Comparisons of trait variances (upper panels) and trait $Q$ values (lower panels) for small variance (solid lines) and large variance (dotted lines) simulations of the phenotype model (equation (18, red lines), the population model (equation (17), blue lines), and the corrected population model (equation 17) with the extra term specified in equation 15, black lines). The small variance simulations are initiated with $\alpha_{1}=40=\alpha_{2}$ and $\beta_{1}=40=\beta_{2}$; the large variance simulations are initiated with $\alpha_{1}=10=\alpha_{2}$ and $\beta_{1}=10=\beta_{2}$.

The differences in timing of the population dynamics appears to be related to the exponential recoveries of the phenotypes and populations from small values. When $P_{2}$ becomes small (compared to the variance) in the population model simulations the timing of its recovery depends upon the "smallness" of the whole population, which recovers when the mean trait value allows it to grow. In the phenotype model, when the phenotype with the mean trait value is unable to grow, because of the trait variance there will be phenotypes that are able to grow. These phenotypes will begin their exponential recovery from small values before the population model. Further, traits with large variances 

grow than the equivalent simulation with small variance, and therefore those phenotypes will recover more rapidly than both the population models and the phenotype models with small variances.

\subsection{Long Simulations}

Here we present the results of 20,000 time step integrations of the population and phenotype models (Figures 18 and 19 ).
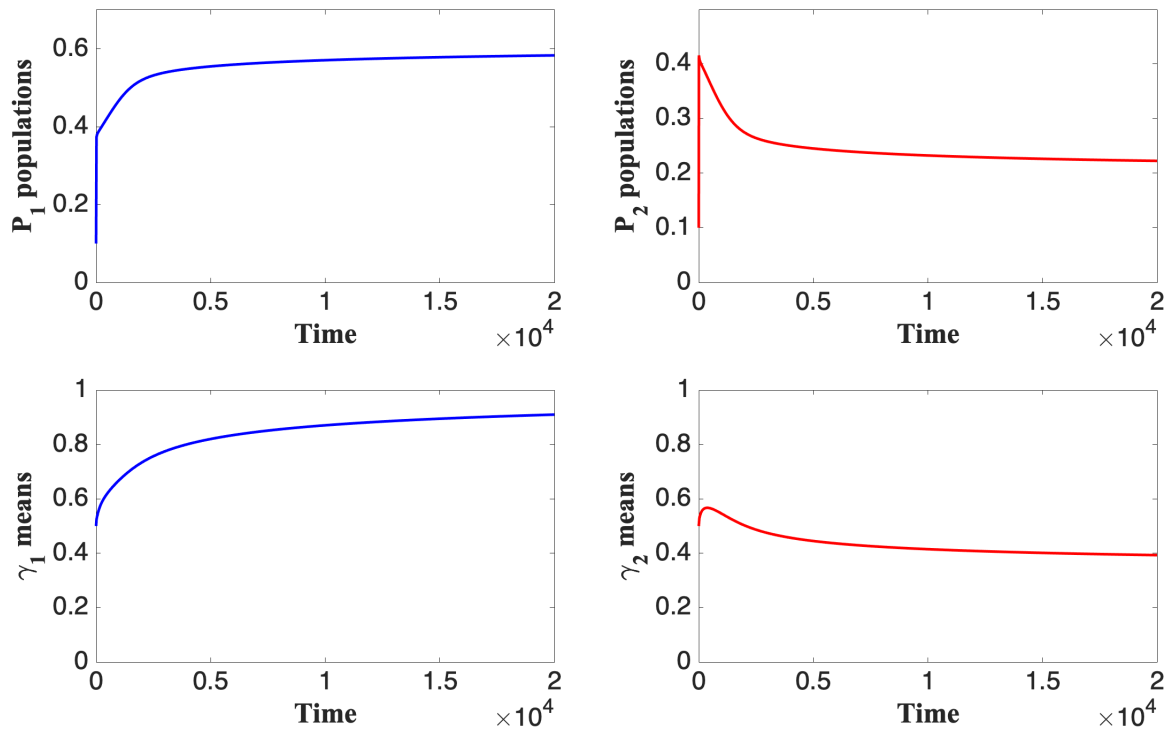

Figure 18: Scenario I: Comparisons of the long time series for population sizes (top panels) and their average trait values (bottom panels) for the phenotype model (equation (18), solid lines) and the population model (equation 17 , dashed lines). Note the dashed lines are not visible as they are coincident with the solid lines. The left panels show $P_{1}$ attributes, and the right panels $P_{2}$ attributes.

\subsection{Bifurcation Analysis of Scenario III}

Scenario III provides an opportunity to demonstrate the advantage of the population modelling approach over the phenotype modelling approach. Dy- 

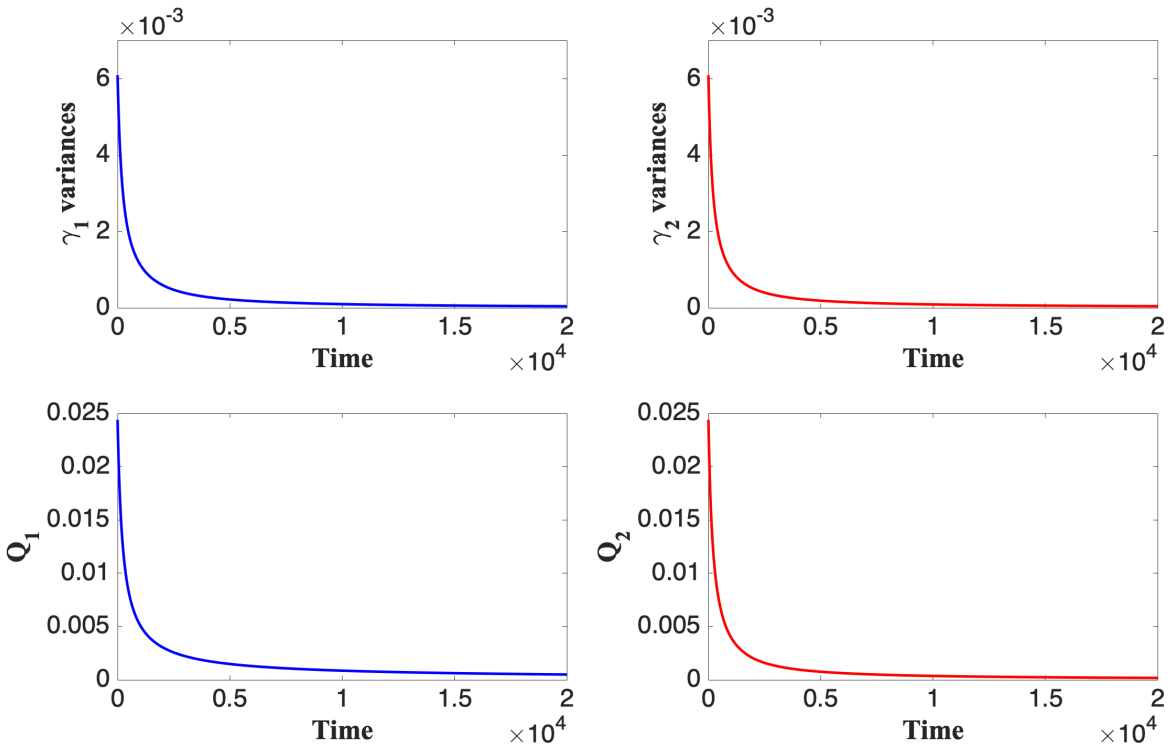

Figure 19: Scenario I: Comparisons of the long time series for trait variances (top panels) and $Q$ values (bottom panels) for the phenotype model (equation 18, solid lines) and the population model (equation (17), dashed lines). Note the dashed lines are not visible as they are coincident with the solid lines. 
namical systems analysis of the population model allows us to see that profound changes to the long-term dynamical properties of the model occur due to changes in the evolutionary space while the ecology appears to be at or near equilibrium.

Figure 20 presents an expanded view of the dynamics of the population model populations in Scenario III, previously shown in Figure 9. In panel (c) of Figure 20 we see that the actual populations simulated by the model (the solid lines) change very little between time 200 and time 300. The ecosystem state has been attracted to the stable equilibrium point on the $P_{1}$ axis and the ecosystem changes slowly here as $f_{1} \approx 0 \approx f_{2}$. (Expressions for the equilibrium points are provided in Appendix 8.1.)

However, calculation of the ecological coexistence equilibrium point (equation (27)) shows that the equilibrium state of the ecology (panel (c), dashed lines) changes significantly in this period. The coexistence equilibrium point, which was located near $P_{1} \approx 0.3, P_{2} \approx 0.1$ for most of the initial stages of the integration, suddenly disappears off to infinity and beyond, as $P_{1}^{*} \rightarrow-\infty$ and $P_{2}^{*} \rightarrow+\infty$, around time 250. However, $P_{1}^{*}$ reappears in the ecospace from $+\infty$, and $P_{2}^{*}$ from $-\infty$, soon after at around time 255. This behaviour is the result of two sequential transcritical bifurcations, in which the coexistence equilibrium point first leaves the ecospace, and then re-enters.

The first transcritical bifurcation occurs at the boundary equilibrium point $\left\{P_{1}^{*}=0, P_{2}^{*}=r_{2} \hat{\gamma}_{2} /\left(r_{2} \hat{\gamma}_{2}-a_{22}\right)\right\}$ when $\hat{\gamma}_{1} \hat{\gamma}_{2}=a_{22} r_{1} / a_{12} r_{2}=0.1904$. The ecospace just prior to this bifurcation is shown in the top left ecospace diagram (panel (a), at time 246), and reveals that the coexistence point has moved close to the $P_{2}$ axis. Panel (d) in Figure 20 plots the locations of the zero isocline intercepts with the $P_{2}$ axis, and identifies that the first transcritical bifurcation (marked by the vertical dashed line) occurs at about time 247, when the axis intercept lines cross, indicating that the axis intercepts have passed through each other. The coexistence equilibrium point leaves the ecospace, and exchanges stability with the boundary equilibrium point, through this bifurcation.

The second transcritical bifurcation occurs at the boundary equilibrium 


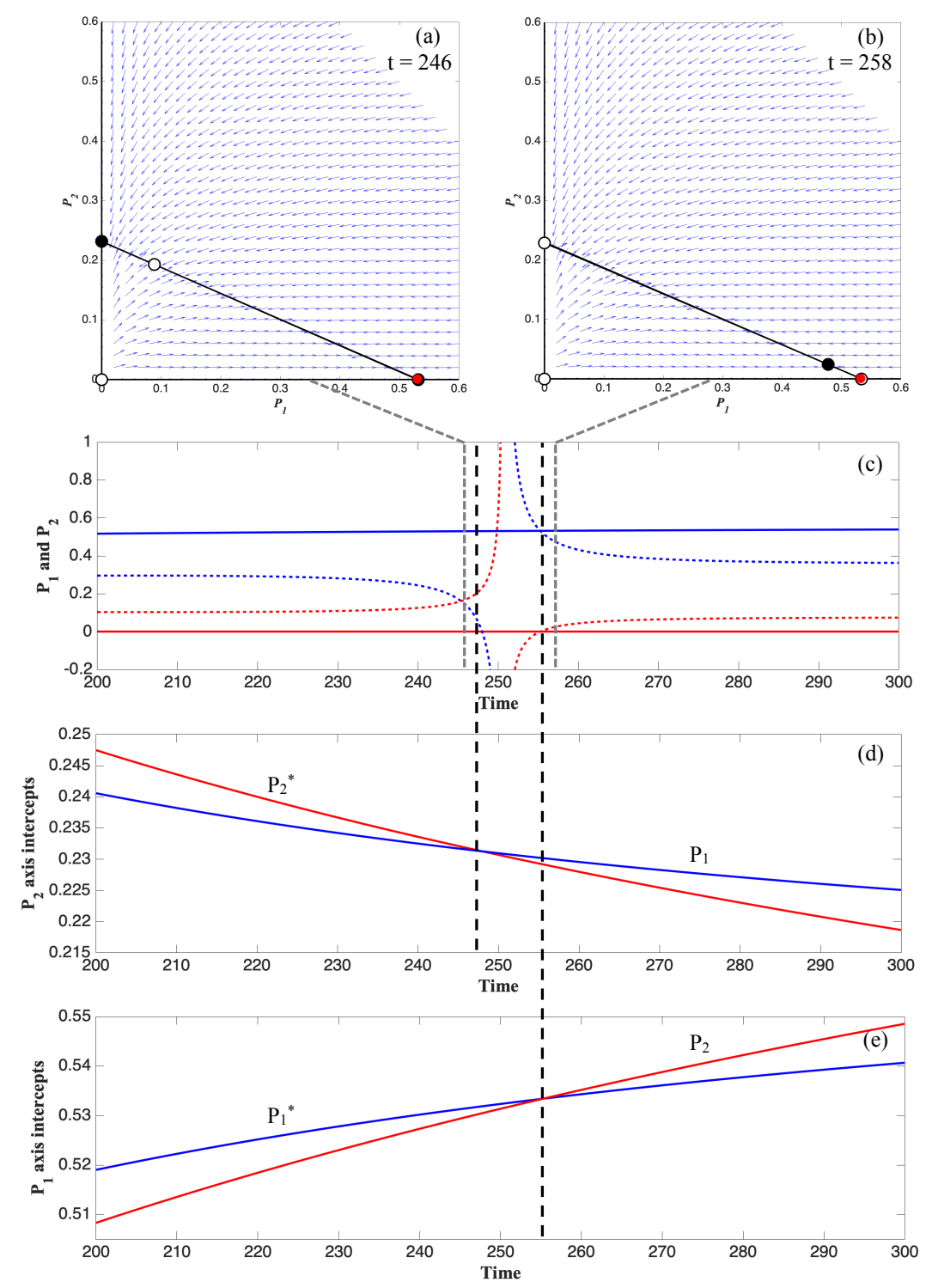

Figure 20: Detail of bifurcation behaviour of Scenario III. In the lower three panels (c, d, and e) the blue lines relate to the $P_{1}$ population and the red lines relate to the $P_{2}$ population. The solid lines in panel (c) show the current state of the populations and the dashed lines show the coexistence ecological equilibrium state for the current values of $\hat{\gamma}_{1}$ and $\hat{\gamma}_{2}$. The top panels ( $\mathrm{a}$ and $\mathrm{b}$ ) show the ecospace diagrams (as per Figures 2 and 4 at times 246, and 258, just before and just after the transcritical bifurcations. The dashed grey lines show where these diagrams are located in time on panel (c). The black dots in (a) and (b) show stable equilibrium points, the white dots unstable equilibrium points, and the red dot shows the current state of the populations. Panel (d) shows the zero isocline intercepts on the $P_{2}$ axis, where the intersection of the lines identifies the first transcritical bifurcation at about time 247. Panel (e) shows the zero isocline intercepts on the $P_{1}$ axis; here the intersection of the lines identifies the second transcritical bifurcation at about time 255. The times that these bifurcations occur in panel (c) are shown by the dashed black lines. 
point $\left\{P_{1}^{*}=r_{1} \hat{\gamma}_{1} /\left(r_{1} \hat{\gamma}_{1}-a_{11}\right), P_{2}^{*}=0\right\}$ when $\hat{\gamma}_{1} \hat{\gamma}_{2}=a_{11} r_{2} / a_{21} r_{1}=0.1894$.

The ecospace just after this bifurcation is shown in the upper right ecospace diagram (panel (b), at time 258), and reveals that the coexistence equilibrium point now lies near the $P_{1}$ axis. Panel (e) in Figure 20 plots the locations of

the zero isocline intercepts with the $P_{1}$ axis, and identifies that the transcritical bifurcation (marked by the vertical dashed line) occurs at about time 255, when the lines cross. The coexistence equilibrium point re-enters the ecospace, and exchanges stability with the boundary equilibrium point at this bifurcation, so that both boundary single-population equilibrium points are unstable, and the internal coexistence equilibrium point is now stable.

Once the coexistence equilibrium point has re-entered the ecospace and is stable, it attracts the ecology away from the boundary point and towards itself. As the system continues to evolve the coexistence equilibrium point moves further into the ecospace, as shown in Figure 4, until it reaches its eco-evolutionary equilibrium point at equation 19 .

Figure 21 presents the initial and final phase plane diagrams for the ecospace (upper panels) and evospace (lower panels) and provides an insight into the dynamics of the system that can be inferred from the population model but not from the phenotype model. The system starts from a state where the internal equilibrium point (27) is unstable (upper left panel), and either $P_{1}$ or $P_{2}$ will drive the other population to extinction in the absence of evolution. Similarly, in the absence of ecological influences, the evolutionary state of the system will come to an internal equilibrium with $\hat{\gamma}_{1}$ and $\hat{\gamma}_{2}$ only slightly changed from their initial conditions (lower left panel).

The eco-evolutionary system however, behaves quite differently. The grey arrows in Figure 21 show which way the associated zero isoclines move in the eco-evolutionary system. The zero isoclines in the ecospace both rotate anticlockwise and translate, with the $f_{1}$ isocline rotating more slowly than the $f_{2}$ isocline, causing the axis intercepts of the isoclines to pass through each other.

1135 Transcritical bifurcations, where equilibrium points collide and exchange stability, occur each time the zero isocline axis intercepts meet, as shown in Figure 

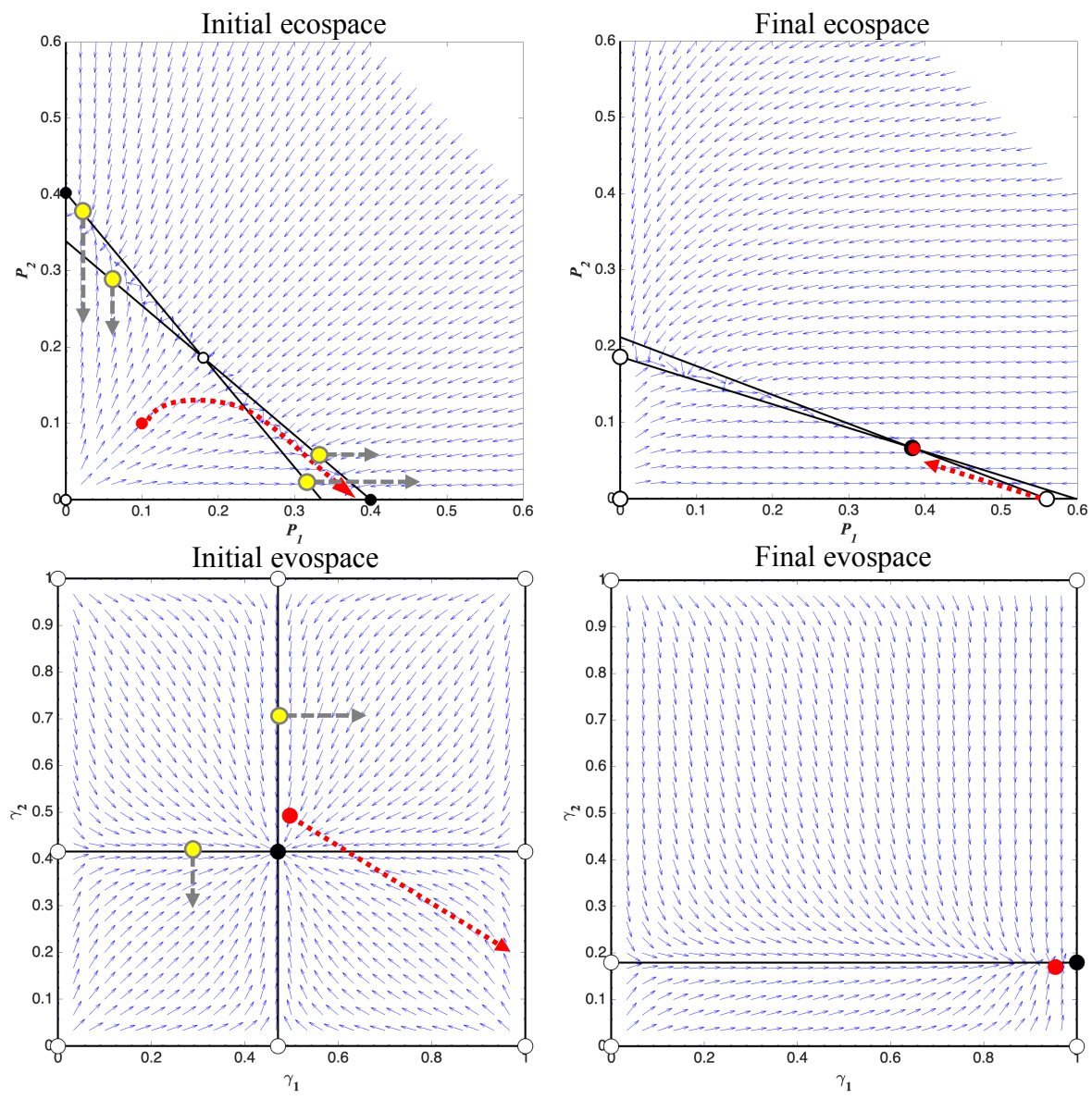

Figure 21: Eco- and evospaces (upper and lower panels respectively) for the initial and final states (left and right panels respectively) of Scenario III. Details as for Figures 2 and 4 Additionally, the grey dashed arrows show which way the initial zero isocline identified by the attached yellow dot will move as the system evolves. Long arrows indicate that the indicated isocline moves faster than those with short arrows. Note the zero isoclines in the ecospace rotate as well as translate, whereas in the evospace, the zero isoclines only translate. The dashed red arrow shows the movement of the equilibrium state of the system. Note that as the zero isoclines move the vector field changes, and the equilibrium point does not follow the vector fields shown. 
20.

As the isoclines move the unstable interior coexistence equilibrium point first moves up and left (Figure 20) until it collides with the $P_{2}^{*}$ point on the ${ }_{1140} P_{2}$ axis. It then leaves the ecospace as the isoclines continue to rotate and remains outside the ecospace, eventually re-appearing below the $P_{1}$ axis. A second transcritical bifurcation occurs when the coexistence equilibrium point re-enters the ecospace through the $P_{1}^{*}$ axis point, whereupon it becomes stable and continues in to the interior of the ecospace, signifying that $P_{2}$ has adapted 1145 to the competitive superiority of $P_{1}$ by limiting $P_{1}$ 's influence upon it (upper right panel).

The zero isoclines in the evospace behave more simply, and just translate as indicated by the grey arrows. The $\hat{\gamma}_{1}$ isocline slides to the right and leaves the evospace, so the only equilibrium points available to the system have $\hat{\gamma}_{1}^{*}=1$. ${ }_{1150}$ Simultaneously the $\hat{\gamma}_{2}$ isocline slides down the evospace until it reaches about $\hat{\gamma}_{2}^{*} \approx 0.18$, that is, the evospace creates a stable equilibrium point at $\left\{\hat{\gamma}_{1}^{*}=\right.$ $\left.1, \hat{\gamma}_{2}^{*}=0.18\right\}$ (lower right panel). 